\title{
Orexin neurons suppress narcolepsy via 2 distinct efferent pathways
}

\author{
Emi Hasegawa, ${ }^{1}$ Masashi Yanagisawa,, ${ }^{2}$ Takeshi Sakurai,1 and Michihiro Mieda1 \\ 1Department of Molecular Neuroscience and Integrative Physiology, Faculty of Medicine, Kanazawa University, Kanazawa, Ishikawa, Japan. \\ 2Department of Molecular Genetics and Howard Hughes Medical Institute, University of Texas Southwestern Medical Center, Dallas, Texas, USA.
}

\begin{abstract}
The loss of orexin neurons in humans is associated with the sleep disorder narcolepsy, which is characterized by excessive daytime sleepiness and cataplexy. Mice lacking orexin peptides, orexin neurons, or orexin receptors recapitulate human narcolepsy phenotypes, further highlighting a critical role for orexin signaling in the maintenance of wakefulness. Despite the known role of orexin neurons in narcolepsy, the precise neural mechanisms downstream of these neurons remain unknown. We found that targeted restoration of orexin receptor expression in the dorsal raphe (DR) and in the locus coeruleus (LC) of mice lacking orexin receptors inhibited cataplexy-like episodes and pathological fragmentation of wakefulness (i.e., sleepiness), respectively. The suppression of cataplexy-like episodes correlated with the number of serotonergic neurons restored with orexin receptor expression in the DR, while the consolidation of fragmented wakefulness correlated with the number of noradrenergic neurons restored in the LC. Furthermore, pharmacogenetic activation of these neurons using designer receptor exclusively activated by designer drug (DREADD) technology ameliorated narcolepsy in mice lacking orexin neurons. These results suggest that DR serotonergic and LC noradrenergic neurons play differential roles in orexin neuron-dependent regulation of sleep/wakefulness and highlight a pharmacogenetic approach for the amelioration of narcolepsy.
\end{abstract}

\section{Introduction}

The neuropeptides orexin A and orexin B (also termed hypocretin-1 and hypocretin-2, respectively) are implicated in feeding, the reward system, and sleep/wake regulation (1). They act on the $\mathrm{G}$ protein-coupled receptors orexin receptor type 1 (OX1R) and OX2R. Degenerative loss of orexin neurons in humans is associated with narcolepsy, a debilitating neurological disorder that provides a unique perspective on the mechanisms of sleep/wakefulness control (2). The syndrome consists of excessive daytime sleepiness that often results in sleep attacks (sudden onset of non-rapid eye movement [NREM] sleep), cataplexy (sudden bilateral skeletal muscle weakening triggered by emotions, without consciousness impairment), hypnagogic hallucinations, and sleep paralysis.

The symptoms of narcolepsy can be divided into 2 independent pathological phenomena $(1,2)$. The first is dysregulation of NREM sleep onset: the inability to maintain a consolidated awake period, characterized by abrupt transitions from wakefulness to NREM sleep. This phenomenon manifests clinically as excessive daytime sleepiness or sleep attacks. The second is dysregulation of rapid eye movement (REM) sleep onset: the pathological intrusion of REM sleep or REM atonia into wakefulness or at sleep onset. It is during these periods that patients may experience cataplexy, hypnagogic hallucinations, and sleep paralysis.

Similarly, mice with targeted deletion of the prepro-orexin gene (orexin ${ }^{-1-}$ mice), mice in which orexin neurons are specifically ablated (orexin/ataxin-3 mice), and mice that lack both orexin receptors $\left(O x 1 r^{-/-} \mathrm{O} \times 2 r^{-/}\right.$mice) display a phenotype strikingly similar to human narcolepsy, with markedly decreased duration of wakefulness episodes during the dark phase (i.e., inability

Conflict of interest: The authors have declared that no conflict of interest exists. Citation for this article: J Clin Invest. 2014;124(2):604-616. doi:10.1172/JCI71017. to maintain a long awake period, or sleepiness), abrupt behavioral arrests with muscle atonia (i.e., potentially cataplexy) that manifest as direct transitions from wakefulness to REM sleep in electroencephalogram/electromyogram (EEG/EMG) recordings, decreased REM sleep latency, and increased REM sleep time during the dark phase (1,3-5).

Orexin neurons are distributed within the lateral hypothalamus and send projections throughout the brain and spinal cord, with particularly dense innervations to nuclei containing monoaminergic and cholinergic neurons that constitute the ascending activating system in the brainstem and the hypothalamus. These include the noradrenergic neurons of the locus coeruleus (LC), the serotonergic neurons of the dorsal raphe nucleus (DR) and median raphe nucleus (MnR), the histaminergic neurons of the tuberomammillary nucleus (TMN), and the cholinergic neurons of the laterodorsal tegmental nucleus (LDT) and pedunculopontine tegmental nucleus (PPT) (1). Application of orexin A directly into the LC (6), TMN (7), or LDT (8) has been reported to increase wakefulness. Furthermore, in vitro slice electrophysiology studies have shown that orexin $\mathrm{A}$ and orexin $\mathrm{B}$ increase the firing rates of monoaminergic neurons in the $\operatorname{LC}(9,10)$, DR $(11,12)$, and TMN $(13-15)$ and cholinergic neurons in the LDT (16). These observations suggest that orexin neurons stabilize wakefulness by regulating these monoaminergic and cholinergic neurons (1).

However, neurons activated by pharmacological application of exogenous orexin may not necessarily be essential for the endogenous mechanisms by which orexin neurons regulate sleep/wakefulness in a physiological condition. In order to identify neurons that are directly activated by endogenous orexins and mediate their wake-stabilizing effect in a natural context, we searched for monoaminergic and cholinergic nuclei in which focal rescue of orexin receptor expression in $\mathrm{O} x 1 r^{-1-} \mathrm{O} \times 2 r^{-/-}$mice ameliorated their narcoleptic phenotype. 


\section{Results}

Search for wake-active monoaminergic/cholinergic nuclei that mediate the suppression of narcoleptic symptoms by orexin neurons. Ox1 $\mathrm{r}^{-1-O} \mathrm{O}_{2 r^{-1-}}$ mice display a phenotype that is strikingly similar to narcolepsy in the dark phase $(1,5)$ : fragmented wakefulness (shorter duration and increased number of wakefulness episodes), cataplexy, shortened REM sleep latency, and excessive REM sleep time. In these mice, projections of orexin neurons to monoaminergic and cholinergic nuclei were retained to an extent similar to that in WT mice (Supplemental Figure 1; supplemental material available online with this article; doi:10.1172/JCI71017DS1). Thus, if the regional restoration of orexin receptors in a certain brain region suppresses narcoleptic symptoms in these mice, that particular region can at least be regarded as one of the important downstream targets of orexin neurons.

Sleep and wakefulness are controlled by a complex network of neurotransmitters and neuromodulators, including glutamate, GABA, monoamines, acetylcholine, and neuropeptides (1). As a first set of candidates in the search for the downstream targets of orexin neurons, we selected the serotonergic DR, noradrenergic LC, histaminergic TMN, and cholinergic PPT for 4 reasons: (a) these monoaminergic and cholinergic nuclei have long been implicated in sleep/wakefulness regulation, (b) they receive dense projections of orexin neurons, (c) good molecular markers are available to evaluate restoration efficiency, and (d) monoaminergic and cholinergic neurons of these nuclei are densely packed in relatively small areas, which makes it comparatively easy to restore orexin receptors in most cells of a particular neuronal population using focal injection of recombinant viral vectors.

We generated recombinant adeno-associated viruses (AAVs) to express either OX1R or OX2R fused to EGFP under the control of the ubiquitous $E F 1 \alpha$ promoter (referred to herein as AAV-EF1 $\alpha / O X 1 R:: E G F P$ and AAV-EF1 $\alpha / O X 2 R:: E G F P)$. We then stereotaxically microinjected AAV-EF1 $\alpha / \mathrm{OX} 1 \mathrm{R}:$ :EGFP into the LC and PPT, or AAV-EF1 $\alpha / O X 2 R:$ EGFP into the TMN and DR, of $O \times 1 r^{-1-} \mathrm{O} \times 2 r^{-1}$ mice (bilaterally for LC, PPT, TMN; at midline for DR) and recorded EEG/EMG to score sleep/wakefulness states. The subtype of orexin receptors expressed was basically determined according to the expression of endogenous orexin receptors in WT mice $(17,18)$. Although the DR expresses both OX1R and OX2R, we assumed that restoration of either one would be sufficient to recover orexin signaling in these cells, as both receptors are $\mathrm{G}_{\mathrm{q}}$-coupled and have caused strong excitatory effects on neurons examined thus far (1).

After completing EEG/EMG recordings, we evaluated the targeted expression of orexin receptors in recorded mice in order to select those with restoration in more than approximately half of cells of interest before scoring the sleep/wake stages (Figure 1). In selected mice, a substantial proportion of nucleispecific monoaminergic or cholinergic neurons expressed OX1R::EGFP or OX2R::EGFP: $72.0 \% \pm 3.2 \%$ of tryptophan hydroxylase-positive $\left(\mathrm{TPH}^{+}\right)$serotonergic neurons in the DR $(n=7), 83.2 \% \pm 3.7 \%$ of tyrosine hydroxylase-positive $\left(\mathrm{TH}^{+}\right)$ noradrenergic neurons in the LC $(n=8), 61.3 \% \pm 5.0 \%$ of histidine decarboxylase-positive $\left(\mathrm{HDC}^{+}\right)$histaminergic neurons in the ventral TMN $(n=4)$, and $79.4 \% \pm 2.9 \%$ of choline acetyltransferase-positive $\left(\mathrm{ChAT}^{+}\right)$cholinergic neurons in the PPT $(n=4)$. OX1R::EGFP or OX2R::EGFP expression was also observed in non-monoaminergic/non-cholinergic neurons within and around the targeted nuclei (Figure 1).
We then analyzed the sleep/wakefulness patterns of selected $O \times 1 r^{-1-} O \times 2 r^{-/-}$mice with satisfactory restoration of orexin receptor expression. As narcoleptic symptoms occur almost exclusively during the dark phase in mice, we hereafter describe sleep/wakefulness in this period. For cataplexy, we first confirmed in several mice that direct transitions from wakefulness to REM sleep in the EEG/EMG data perfectly correlated with episodes of abrupt immobility in the video data. Therefore, for the rest of the mice, we simply counted the direct transitions from wakefulness to REM sleep. We refer to these herein as cataplexy-like episodes, according to Scammell et al. (19) (see Methods).

Restoration of OX2R in the DR $\left(O \times 1 r^{-1-} O x 2 r^{-1-+D R-O X 2 R}\right.$ mice) completely prevented cataplexy-like episodes, while the fragmentation of wakefulness was unaffected (Figure 2, A and C). Excessive REM sleep time was partially but significantly ameliorated, while REM sleep latency was not significantly increased, in $\mathrm{O} \times 1 r^{-1-} \mathrm{O} \times 2 r^{-1-}+\mathrm{DR}-\mathrm{OX} 2 \mathrm{R}$ versus control $\mathrm{O} \times 1 r^{-1-} \mathrm{O} \times 2 r^{-1}$ mice (Figure $2 \mathrm{~B}$ ). The mean duration and number of wakefulness episodes, as well as the total wakefulness time, did not significantly differ between groups (Figure 2C and Supplemental Figure 2B).

In clear contrast to the $\mathrm{DR}$, restoration of OX1R in the LC (Ox $1 r^{-1-}-0 \times 2 r^{-1-+}$ LC-OX1R mice) significantly improved the maintenance of wakefulness and NREM sleep (Figure 2C and Supplemental Figure 2A). Wakefulness episode duration was significantly increased and the number of wakefulness episodes significantly reduced in $\mathrm{O} x 1 r^{-/-} \mathrm{O} \times 2 r^{-1-}+\mathrm{LC}-\mathrm{OX} 1 \mathrm{R}$ versus control $O \times 1 r^{-1-} \mathrm{O} \times 2 r^{-1-}$ mice (Figure 2C). The total time of wakefulness was not significantly different between groups (Supplemental

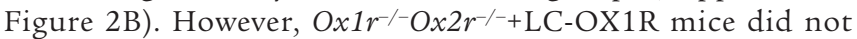
exhibit a significant change in the frequency of cataplexy-like episodes and excess REM sleep time, while it partially but significantly ameliorated shortened REM sleep latency, compared with $\mathrm{O} \times 1 r^{-1-} \mathrm{O} \times 2 r^{-1-}$ controls (Figure 2, A and B). Targeted restoration of OX1R in the PPT or OX2R in the TMN of Ox1 $1 r^{-1-} \mathrm{O} 2 r^{-1-}$ mice $\left(O x 1 r^{-/-} O x 2 r^{-1-}+\mathrm{PPT}-\mathrm{OX} 1 \mathrm{R}\right.$ and $O \times 1 r^{-/-} \mathrm{O} \times 2 r^{-/-}+\mathrm{TMN}-\mathrm{OX} 2 \mathrm{R}$, respectively) did not ameliorate either the fragmentation of wakefulness or cataplexy-like episodes (Figure 2).

As described above, orexin receptor expression was driven by the ubiquitous $E F 1 \alpha$ promoter, resulting in their additional expression in the vicinity of the DR or LC (Figure 1). In addition, multiple types of neurons endogenously express OX1R and/or OX2R in these nuclei in WT mice (18). Indeed, only $62.2 \% \pm 2.8 \%$ of EGFP ${ }^{+}$ neurons were $\mathrm{TPH}^{+}$in $\mathrm{O} x 1 r^{-/} \mathrm{O} \times 2 r^{--}+\mathrm{DR}-\mathrm{OX} 2 \mathrm{R}$ mice $(n=7)$, while

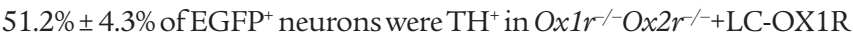
mice $(n=7)$. We next performed Pearson correlation analysis in order to examine which neuronal population has a linear doseresponse relationship between the magnitude of amelioration of narcoleptic symptoms and the number of cells with restoration of orexin receptor expression (Figure 3). For this purpose, we included EEG/EMG and histological data of mice with off-target restoration (i.e., restoration by orexin receptor in DR serotonergic neurons or in LC noradrenergic neurons, which were found to be less than $50 \%$ and eliminated from the initial analyses). As shown in Figure 3A, inhibition of cataplexy-like episodes was highly correlated with the number of EGFP+ $\mathrm{TPH}^{+}$cells in the DR, but was not significantly correlated with the number of $\mathrm{EGFP}^{+} \mathrm{TPH}^{-}$cells within the DR, in the region lateral to the $\mathrm{DR}$, or in the ventrolateral part of the periaqueductal gray matter (vlPAG), which has previously been reported to suppress REM sleep (20). Similarly, the duration of wakefulness episodes was highly correlated with the number of 
A DR

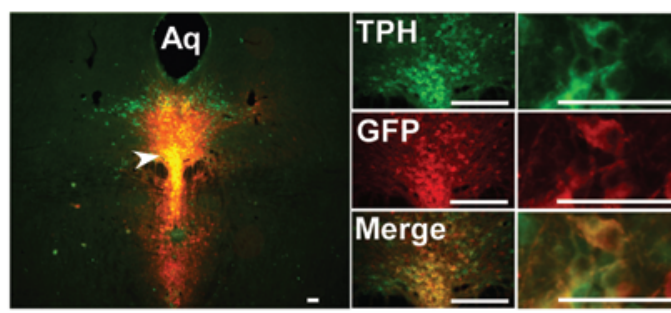

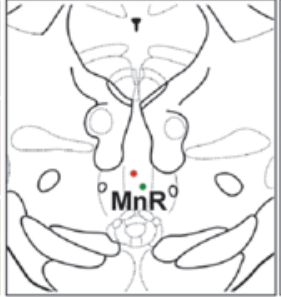

Bregma $-4.04 \mathrm{~mm}$

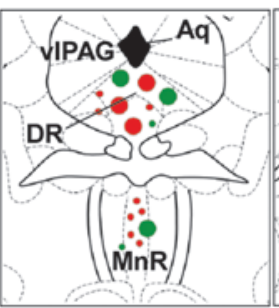

Bregma $-4.60 \mathrm{~mm}$

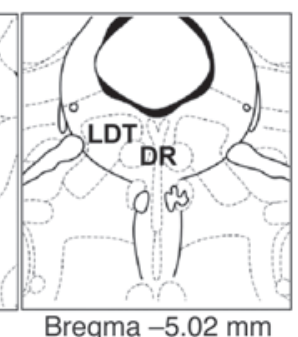

Bregma $-5.02 \mathrm{~mm}$

B
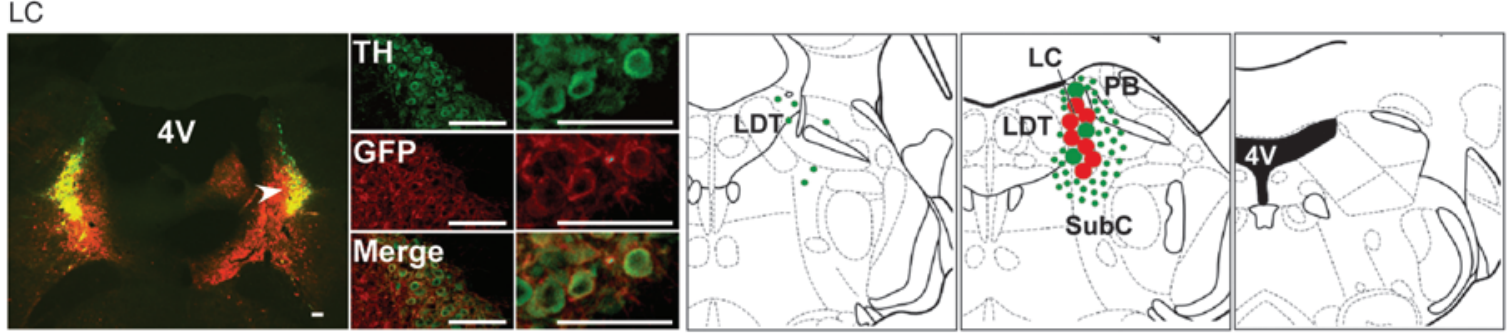

Bregma $-5.20 \mathrm{~mm}$

Bregma $-5.34 \mathrm{~mm}$

Bregma $-5.80 \mathrm{~mm}$

C TMN
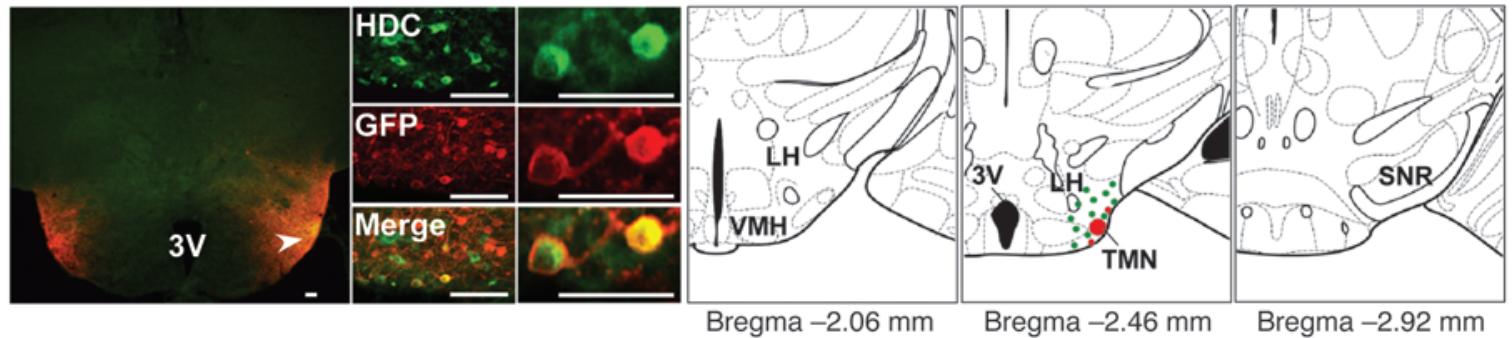

D
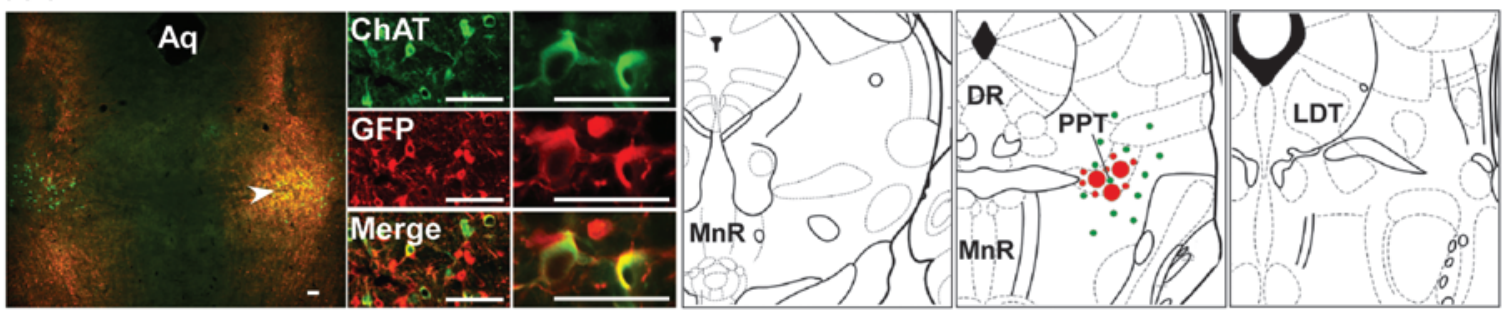

Bregma $-4.04 \mathrm{~mm}$

Bregma $-4.48 \mathrm{~mm}$

Bregma $-4.96 \mathrm{~mm}$

EGFP(+)/neuron type-specific marker(-)

100 cells $\quad 10$ cells

EGFP(+)/neuron type-specific marker(+)

100 cells

- 10 cells

Figure 1

Region-specific restoration of orexin receptor expression in $\mathrm{O} \times 1 r^{-1-} \mathrm{O} \times 2 r^{-1-}$ mice. Coronal brain sections prepared from $\mathrm{O} \times 1 r^{-1-O} \mathrm{O} 2 r^{-1-}$ mice with targeted injection of AAV-EF1 $\alpha / O X 1 R:: E G F P(B$ and $\mathbf{D})$ or AAV-EF1 $\alpha / O X 2 R:: E G F P(A$ and $\mathbf{C})$ were double-stained with anti-GFP (red) and the neuronal type-specific marker (green) antibodies TPH (A), TH (B), HDC (C), and ChAT (D). Regions denoted by white arrowheads are shown at higher magnification. Schematics show the spread of OX1R::EGFP or OX2R::EGFP expression. Mean numbers of EGFP+ cells are shown by green or red circles, indicative of the absence or presence, respectively, of the neuronal type-specific marker. For bilaterally injected areas, only 1 side was shown. $3 \mathrm{v}$, third ventricle; $4 \mathrm{v}$, fourth ventricle, Aq, aqueduct; SNR, substantia nigra pars reticulata; $\mathrm{VMH}$, ventromedial hypothalamus. Scale bars: $100 \mu \mathrm{m}$.

$\mathrm{EGFP}^{+} \mathrm{TH}^{+}$cells in the LC, but was not significantly correlated with $\mathrm{EGFP}^{+} \mathrm{TH}^{-}$cell number within the LC or in any surrounding area, such as the subcoeruleus (SubC) region or the parabrachial nucleus (PB) (Figure 3B). Taken together, these results suggest that restoration of orexin signaling in the DR and in the LC may inhibit cataplexy-like episodes and fragmentation of wakefulness, likely mediated by serotonergic and noradrenergic neurons, respectively.

Restoration of orexin receptors in DR or LC using neuron type-selective promoters. We next aimed to restore the expression of orexin receptors in a neuronal type-selective manner in these areas. In the 
A

Cataplexy-like episode

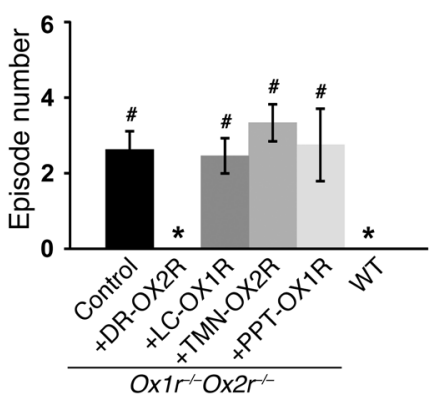

B
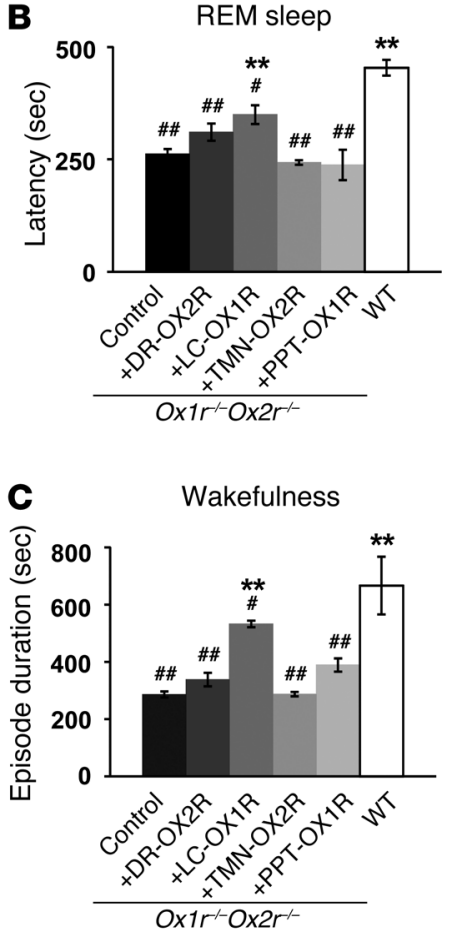

Cataplexy-like episode
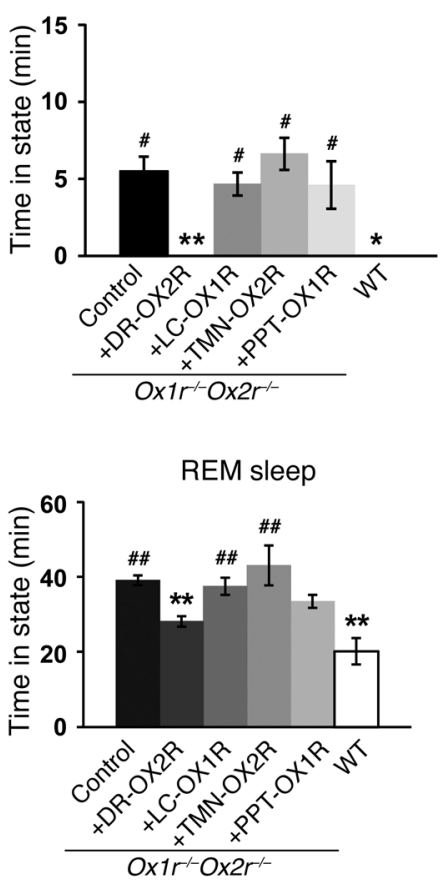

Wakefulness

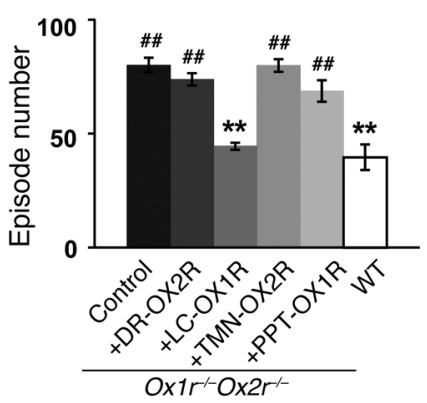

Figure 2

Restoration of orexin receptor expression in the DR and LC inhibits cataplexy-like episodes and consolidates wakefulness, respectively. Control Ox1 $1 r^{-1-O} 2 r^{-1-}(n=16), O \times 1 r^{-1-O} 2 r^{-1-}+\mathrm{DR}-\mathrm{OX} 2 \mathrm{R}(n=7)$, Ox1 $r^{-1-O} 22^{-1-}+$ LC-OX1R $(n=8), O \times 1 r^{-1-O} 2 r^{-1-}+$ TMN-OX2R $(n=4)$,

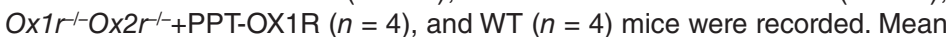
values of 3 nights' recordings are shown. $O \times 1 r^{-1-O} \times 2 r^{-1}$ controls were injected with AAV-CAG/EGFP into the DR or LC, AAV-Pet1/ChR2::EYFP into the DR, or AAV-PRSx8/ChR2::EYFP into the LC. (A) Number of and time spent in cataplexy-like episodes. (B) REM sleep latency and time spent in REM sleep. (C) Duration and number of wakefulness episodes during the dark phase.

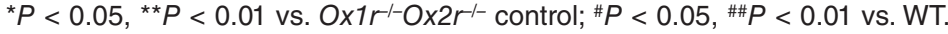
Values are mean \pm SEM.

DR, serotonergic neurons express both OX1R and OX2R (18). To selectively target the expression of OX2R in serotonergic neurons, we generated AAV-Pet1/OX2R::EYFP, an AAV vector encoding OX2R::EYFP driven by a $1.8-\mathrm{kb}$ promoter of the Pet 1 gene (21), which encodes a serotonergic-specific transcription factor. When AAV-Pet1/OX2R::EYFP was microinjected into the DR $\left(O \times 1 r^{-1-O} \times 2 r^{-1-}+5\right.$ HT-OX2R mice; $\left.n=6\right), 88.9 \% \pm 2.5 \%$ of neurons expressing OX2R::EYFP in the DR were also $\mathrm{TPH}^{+}$, whereas $11.1 \%$ $\pm 2.5 \%$ were $\mathrm{TPH}^{-}$, non-serotonergic neurons (Figure 4A). Thus, selectivity of OX2R expression in serotonergic neurons was substantially higher in $\mathrm{O} x 1 r^{-1-} \mathrm{O} \times 2 r^{--}+5 \mathrm{HT}-\mathrm{OX} 2 \mathrm{R}$ than $\mathrm{O} x 1 r^{-/} \mathrm{O} x 2 r^{--}+\mathrm{DR}-\mathrm{OX} 2 \mathrm{R}$ mice (in which $62.2 \% \pm 2.8 \%$ of $\mathrm{EGFP}^{+}$neurons were also $\mathrm{TPH}^{+}$). The proportion of DR serotonergic neurons expressing OX2R::EYFP was comparable in $O x 1 r^{-/-} O \times 2 r^{-/-}+5 \mathrm{HT}-\mathrm{OX} 2 \mathrm{R}$ and Ox $1 r^{-/} \mathrm{O} \times 2 r^{-1-+D R-O X 2 R m i c e}(75.1 \% \pm 2.2 \%$ and $72.0 \% \pm 3.2 \%$ of $\mathrm{EYFP}^{+}$neurons, respectively, were also $\mathrm{TPH}^{+}$).

The vlPAG and the lateral pontine tegmentum (LPT)/ deep mesencephalic reticular nucleus (DPMe), located adjacent to the DR, inhibit REM sleep $(20,22,23)$. However, few cells in these regions expressed OX2R::EYFP in Ox1 $r^{-1-} \mathrm{O} \times 2 r^{-1-}+5 \mathrm{HT}-\mathrm{OX} 2 \mathrm{R}$ mice (Figure 4B). Furthermore, wake-active dopaminergic neurons in the ventral part of the periaqueductal gray matter (vPAG) and a part of the adjacent DR have been implicated in promoting wakefulness (24). Nevertheless, we detected OX2R::EYFP in a very small population of those $\mathrm{TH}^{+}$dopaminergic neurons of Ox $1 r^{-1-} \mathrm{O} \times 2 r^{-1-}+5 \mathrm{HT}-\mathrm{OX} 2 \mathrm{R}$ mice: $1.3 \% \pm 0.5 \%$ of $\mathrm{EYFP}^{+}$ neurons were also $\mathrm{TH}^{+}(3.7 \pm 1.1$ cells; Figure $4 \mathrm{C})$. It is therefore unlikely that the effects of OX2R restoration in $\mathrm{O} \times 1 r^{-1-} \mathrm{O} \times 2 r^{-1-}$ 5HT-OX2R mice are mediated by expression leaked in vlPAG/LPT or vPAG dopaminergic neurons. The relatively serotonergic-selective $\mathrm{OX} 2 \mathrm{R}$ restoration in $O x 1 r^{-1-O} 2 r^{-1-5 H T-O X 2 R}$ mice mimicked the effects of nonselective restoration achieved in $\mathrm{O} \times 1 r^{-/-} \mathrm{O} \times 2 r^{-1}+\mathrm{DR}-\mathrm{OX} 2 \mathrm{R}$ mice (Figure 5 and Supplemental Figure 3). Suppression of cataplexy-like episodes was nearly complete in

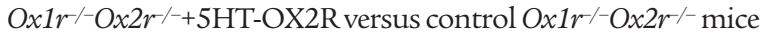
(Figure 5A). However, excessive REM sleep time and shortened REM sleep latency were not significantly ameliorated in these mice (Figure 5B), which suggests that regulation of cataplexy and REM sleep are at least partially different.

In the LC, noradrenergic neurons express OX1R exclusively in WT mice (18). To selectively target OX1R expression in noradrenergic neurons, we generated an AAV vector encoding OX1R::EYFP under the control of the PRS $x 8$ promoter (AAV-PRSx8/OX1R::EYFP; ref. 25). This promoter has been used for the same purpose in previous studies (26-28) and contains a minimal promoter and 8 copies of a promoter sequence from the cis-regulatory region of the dopamine $\beta$-hydroxylase $(D B H)$ gene that binds the Phox $2 \mathrm{a} / \mathrm{b}$ transcription factors. These factors are expressed in and critical for noradrenergic neurons, but they are also expressed in several populations of non-noradrenergic neurons in the brainstem. When AAV-PRSx8/OX1R::EYFP

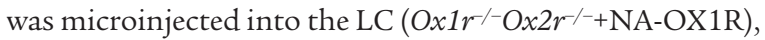
OX1R::EYFP was efficiently expressed in noradrenergic neurons of the LC, but additionally expressed in many nonnoradrenergic neurons in the adjacent areas, including the SubC region and PB (Figure 4, D-G, and ref. 26). Overall, $60.1 \% \pm 6.7 \%$ of neurons expressing OX1R::EYFP were also $\mathrm{TH}^{+}$ $(n=6)$. The proportion of LC noradrenergic neurons expressing

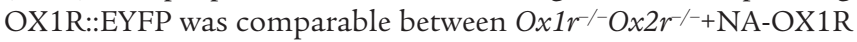
and $O x 1 r^{-1-O} \times 2 r^{-1-}+$ LC-OX1R mice $(88.6 \% \pm 2.6 \%$ and $83.2 \% \pm 3.7 \%$ of $\mathrm{EYFP}^{+}$neurons, respectively, were also $\left.\mathrm{TH}^{+}\right)$.

OX1R restoration in $O x 1 r^{-1-} O x 2 r^{-1-+N A-O X} 1 \mathrm{R}$ mice mimicked the effects of nonselective restoration achieved in Ox1 $r^{-1-O} \times 2 r^{-1+L C-O X 1 R}$ mice (Figure 5). The duration of wakeful- 
A

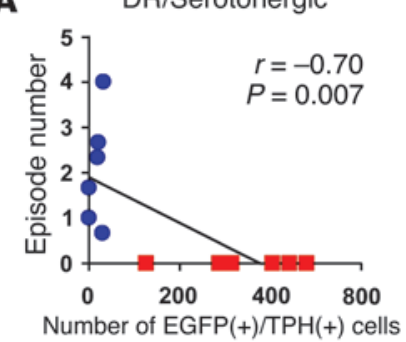

DR/Non-serotonergic

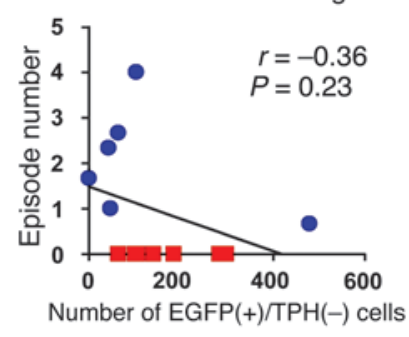

VIPAG

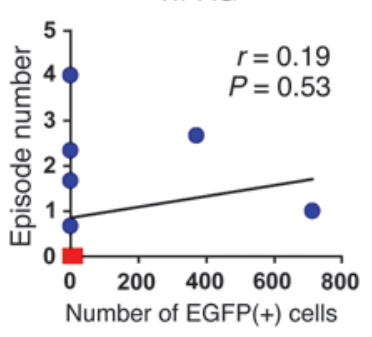

Lateral

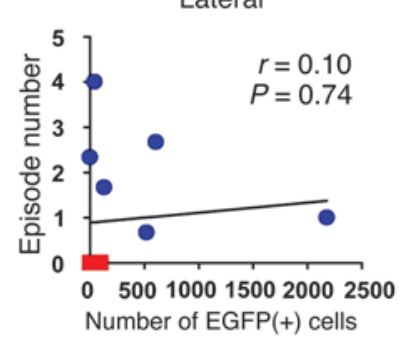

B LC/Noradrenergic
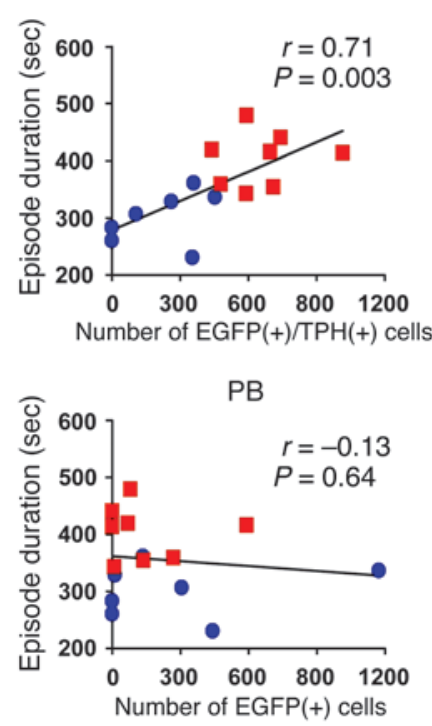

LC/Non-noradrenergic
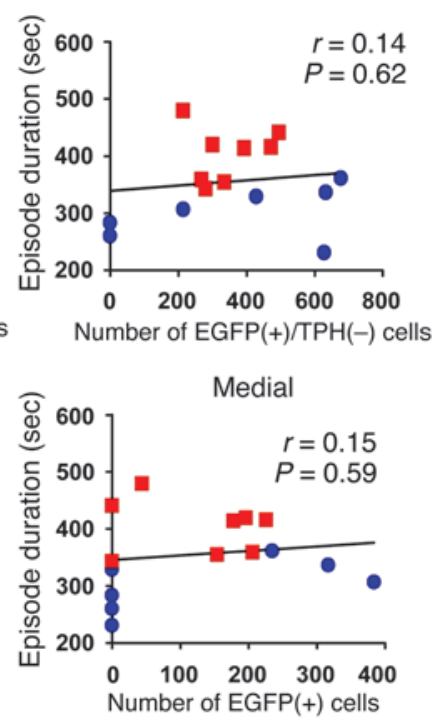

- Successfully targeted

- Unsuccessfully targeted

Figure 3

Suppression of narcoleptic symptoms is correlated with the number of serotonergic or noradrenergic neurons with orexin receptor in the DR or LC. (A) Correlation between number of cataplexy-like episodes and number of EGFP+ cells in TPH+ DR, TPH- DR, vIPAG, or the region lateral to the $\mathrm{DR}$ and $\mathrm{MnR}$ (including the paratrochlear nucleus and pontine reticular nucleus) of $\mathrm{O} \times 1 \mathrm{r}^{-1} \mathrm{O} \times 2 \mathrm{r}^{-1}+\mathrm{DR}-\mathrm{OX} 2 \mathrm{R}$ mice (Successfully targeted) and those with off-target restoration (Unsuccessfully targeted). (B) Correlation between wakefulness episode duration and number of EGFP+ cells in $^{2}$ $\mathrm{TH}^{+} \mathrm{LC}, \mathrm{TH}^{-} \mathrm{LC}$, or regions lateral (including PB), medial, or ventral (including SubC) to the LC of Ox1 $r^{-1} \mathrm{O} \times 2 r^{-1}+\mathrm{LC}-\mathrm{OX} 1 \mathrm{R}$ mice and those with off-target restoration. Pearson $r, P$ values, and regression lines are shown.

ness episodes was significantly increased and the number of wakefulness episodes significantly decreased in $O x 1 r^{-1-O} \times 2 r^{-1+N A-O X 1 R}$ versus control Ox1 $r^{-}-\mathrm{O} \times 2 r^{--}$mice, but these levels were still significantly different from those of WT mice (Figure 5C). Importantly, these parameters of wakefulness were not significantly different from those in $O x 2 r^{-/}$mice (29), which were intermediate between the parameters in $O \times 1 r^{-1-O} \times 2 r^{-1-}$ controls and WT mice, as reported previously $(1,30)$. In WT mice, most wakefulness occurred in episodes longer than 32 minutes (Figure 5D), consistent with a value reported previously (30). In contrast, in $\mathrm{O} \times 1 r^{\prime-O} \times 2 r^{\prime-}$ controls, most wakefulness occurred in episodes of intermediate length, and a marginal proportion of wakefulness occurred in long episodes ( $>32$ minutes), confirming the fragmentation of wakefulness in these mice. The proportion of wakefulness that occurred in long episodes in $O \times 1 r^{-/ O} \times 2 r^{-1-+N A-O X 1 R}$ mice was intermediate between that of $O x 1 r^{-}-O x 2 r^{-/}$control and WT mice. This parameter was not significantly different from that

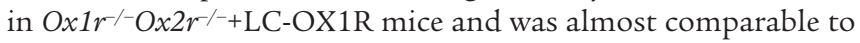
that in $O x 2 r^{-}$mice, but was slightly and significantly reduced. In contrast, none of the parameters that reflected dysregulated REM sleep were significantly improved in $O x 1 r^{/-O} \times 2 r^{/-+N A-O X 1 R}$ versus control Ox1r/-Ox2r/- mice (Figure 5, A and B).

As described above, OX1R::EYFP expression in Ox1 $r^{-1-O} \times 2 r^{-1-N A}-O X 1 \mathrm{R}$ mice spread into areas adjacent to the LC. To evaluate the contribution of such leaked expression to the effect on fragmented wakefulness, we scored the EEG/EMG data of mice with off-target restoration (Ox1 $r^{--O} 2 r^{-/-}$non-NA-OX1R mice), which have similar numbers of EYFP ${ }^{+} \mathrm{TH}^{-}$cells, but few EYFP ${ }^{+} \mathrm{TH}^{+}$cells (Supplemental Figure 4A). In addition, as we detected a considerable number

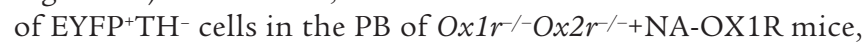
a region that was recently demonstrated to be critical for cortical arousal (31), we focally injected AAV-EF1 $\alpha / O X 1 R:: E G F P$ in the PB of Ox1 $1 r^{-1-O} \times 2 r^{-/}$mice (Ox1r/-Ox2r/-PB-OX1R mice) and analyzed their EEG/EMG as another control (Supplemental Figure 4B). Importantly, both $O x 1 r^{-1-O} \times 2 r^{-1+\text { non-NA-OX1R }}$ and $O x 1 r^{-1-O} \times 2 r^{-1-P B}-\mathrm{OX} 1 \mathrm{R}$ mice demonstrated fragmented wakefulness comparable to that of control $\mathrm{O} \times 1 r^{-1-} \mathrm{O} \times 2 r^{-1}$ mice (Supplemental Figure 4, C-H). Furthermore, wakefulness duration was strongly correlated with the number of $\mathrm{EYFP}^{+} \mathrm{TH}^{+}$cells 
$\mathbf{A}_{\mathrm{DR}}$
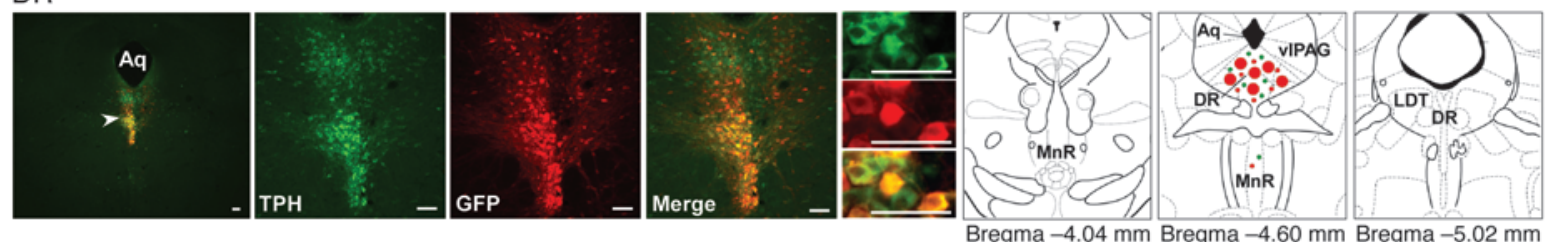

B

VIPAG

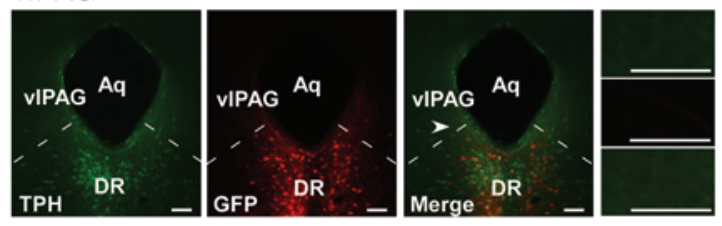

C

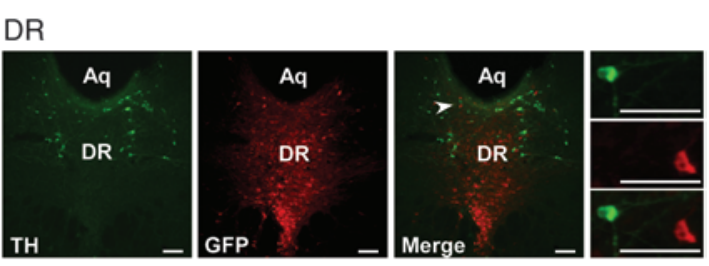

D
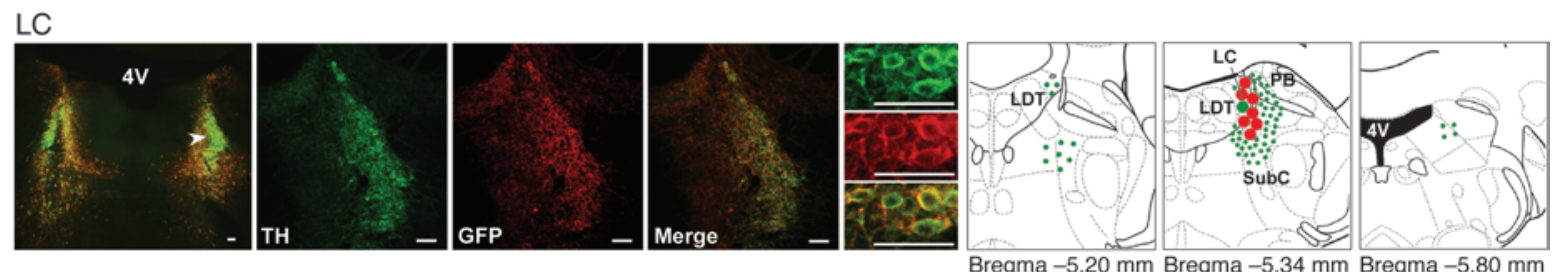

E
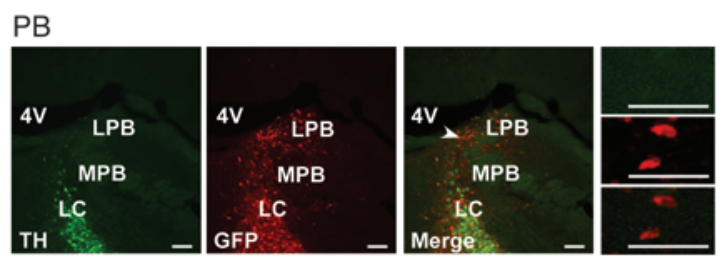

F

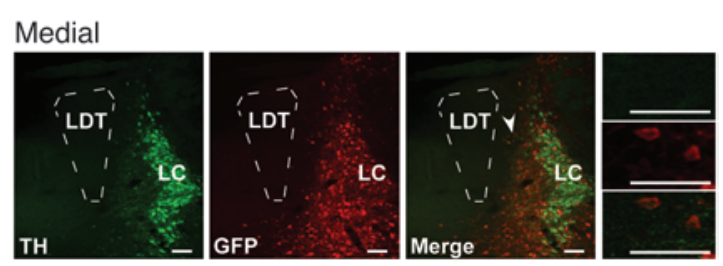

G

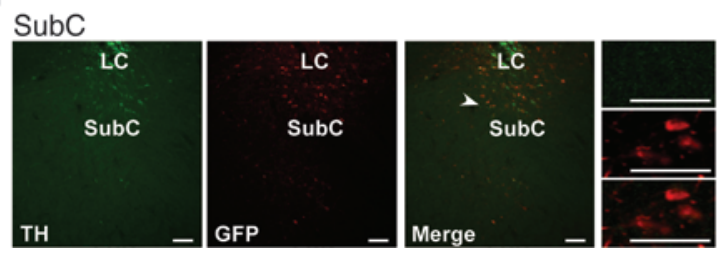
EYFP(+)/neuron type-specific maker(-)
- 100 cells $\quad 10$ cells
$\operatorname{EYFP}(+) /$ neuron type-specific maker(+)
- 100 cells $\quad 10$ cells

Figure 4

Restoration of orexin receptors in the DR or LC of Ox1 $1 r^{-1-O} x_{2} r^{-1-}$ mice using neuron type-selective promoters. (A-C) Coronal brain sections from $\mathrm{O} \times 1 r^{-1-O} \mathrm{O} 2 r^{-1-}+5 \mathrm{HT}-\mathrm{OX} 2 \mathrm{R}$ mice (with targeted injection of AAV-Pet1/OX2R::EYFP) were double-stained with anti-GFP antibody (red) and either

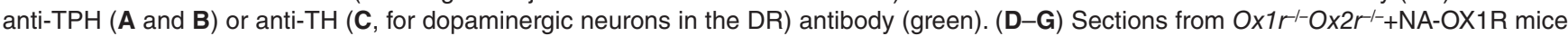
(with AAV-PRSx8/OX1R::EYFP injection) were double-stained with anti-GFP (red) and anti-TH (green) antibodies. Leaked expression of OX1R::EYFP in regions lateral (E, including PB), medial (F), and ventral (G, including SubC) to the LC (D) is shown. Regions denoted by white arrowheads are shown at higher magnification. Schematics show the spread of OX1R::EYFP or OX2R::EYFP expression. Mean numbers of $\mathrm{EGFP}^{+}$cells are shown by green or red circles, indicative of the absence or presence, respectively, of the neuronal type-specific marker. For LC, only 1 side was shown. LPB, lateral PB; MPB, medial PB. Scale bars: $100 \mu \mathrm{m}$.

in the LC, but not with the number of $\mathrm{EYFP}^{+} \mathrm{TH}^{-}$cells in any surrounding area (Supplemental Figure 5A). We also considered the possibility that restoration of orexin signaling in neurons expressing neuropeptide S (NPS) contributes to the consolidation of wakefulness in $O x 1 r^{-1-} \mathrm{O} \times 2 r^{-1-+N A-O X} 1 \mathrm{R}$ mice: NPS promotes wakefulness and is expressed in a small cluster of neurons localized in close proximity to the LC (32). For this purpose, we detected OX1R::EYFP mRNA and NPS mRNA simultaneously using double in situ hybridization. In $O \times 1 r^{-/} \mathrm{O} \times 2 r^{-/-} \mathrm{NA}-\mathrm{OX} 1 \mathrm{R}$ mice, $38.8 \% \pm 11.2 \%$ of $N P S^{+}$cells in this area were also $E Y F P^{+}$. However, the number of $E Y F P^{+} N P S^{+}$cells was small and not significantly correlated with the duration of wakefulness episodes (Supplemental Figure 5B), which suggests that the contribution of NPS neurons is unlikely. Together, these results suggest that 
A
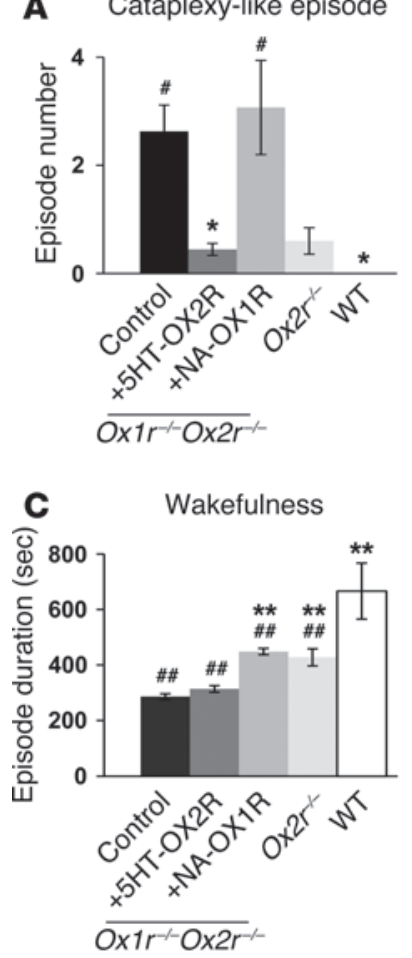

Cataplexy-like episode

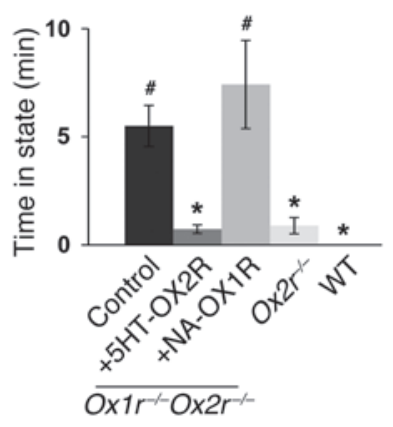

Wakefulness

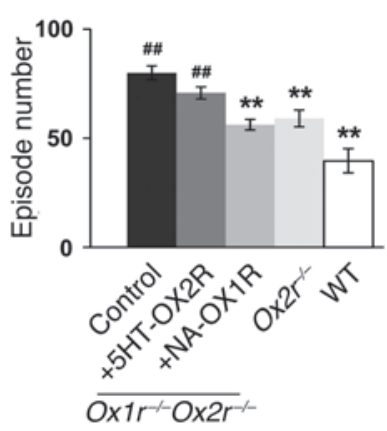

B

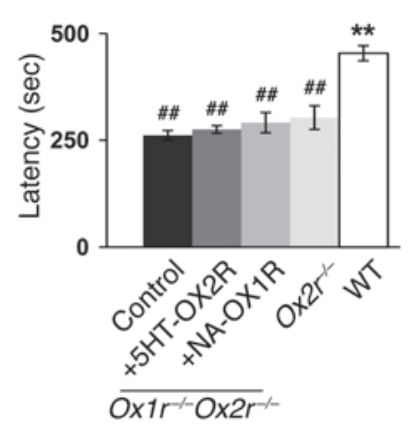

REM sleep

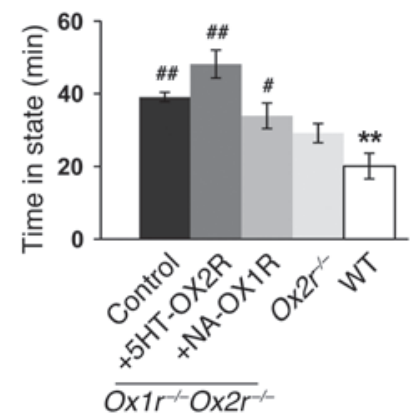

D

Dakefulness

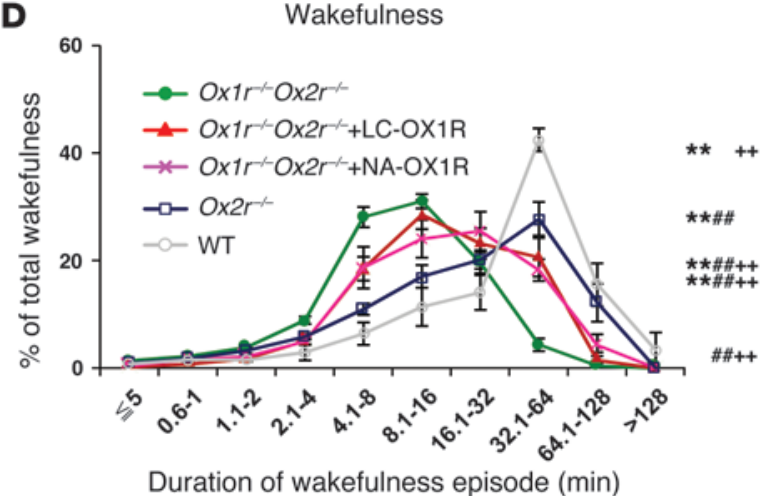

\section{Figure 5}

Restoration of orexin receptor expression selectively in DR serotonergic and LC noradrenergic neurons suppresses cataplexy-like episodes and

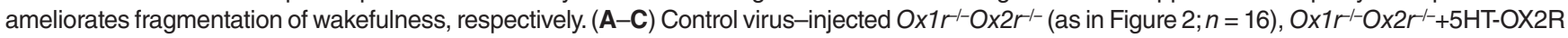
$(n=6), O \times 1 r^{-1-O} 2 r^{-1-}+\mathrm{NA}-\mathrm{OX} 1 \mathrm{R}(n=5), O \times 2 r^{-1-}(n=5)$, and WT $(n=4)$ mice were recorded. Mean values of 3 nights' recordings are shown. Data from Figure 2 are included for comparison. (A) Number of and time spent in cataplexy-like episodes. (B) REM sleep latency and time spent in REM sleep. (C) Duration and number of wakefulness episodes during the dark phase. ${ }^{*} P<0.05,{ }^{* \star} P<0.01$ vs. $O x 1 r^{-1-} O \times 2 r^{-1}$ control; ${ }^{\#} P<0.05$, $\# \# P<0.01$ vs. WT. (D) Time-weighted frequency histograms of wakefulness duration in control $O \times 1 r^{-1-O} 2 r^{-1-}(n=16), O \times 1 r^{-1-O}-2 r^{-1-}+L C-O X 1 R$ $(n=8), O \times 1 r^{-1-O} 2 r^{-1-}+\mathrm{NA}-\mathrm{OX} 1 \mathrm{R}(n=5), O \times 2 r^{-1-}(n=5)$, and WT $(n=4)$ mice, showing the wakefulness that occurred in episodes of each length as a percentage of total wakefulness in the dark phase. ${ }^{\star \star} P<0.001$ vs. $O \times 1 r^{-1-O x} 2 r^{-1-}$ control; ${ }^{\# \#} P<0.001$ vs. WT; ${ }^{++} P<0.001$ vs. Ox2 $r^{-1}$, $2-$ way repeated-measures ANOVA. Values are mean \pm SEM.

the orexinergic regulation of LC noradrenergic neurons may markedly consolidate wakefulness, to an extent comparable to that in $O x 2 r^{-1-}$ mice.

Pharmacogenetic activation of $D R$ serotonergic and $L C$ noradrenergic neurons in narcoleptic mice. We next examined whether artificial activation of DR serotonergic and LC noradrenergic neurons improves narcoleptic symptoms in orexin/ataxin-3 mice (4). In these mice, orexin neurons degenerate postnatally due to the transgenic expression of a truncated Machado-Joseph disease gene product, mimicking the pathophysiological condition of human narcolepsy. For this purpose, we used designer receptors exclusively activated by designer drugs (DREADD) technology, in which muscarinic receptors are mutated so that their ability to bind natural ligands is lost, while nanomolar potency is gained for the otherwise pharmacologically inert ligand clozapine-N-oxide (CNO) $(33,34)$. The stimulatory DREADD, designated "hM3Dq," couples through the $\mathrm{G}_{\mathrm{q}}$ pathway to depolarize neurons. Here, we generated AAV vectors encoding hM3Dq under the control of the Pet 1 or $P R S x 8$ promoter (AAV-Pet1/hM3Dq or AAV-PRSx8/hM3Dq).

When AAV-Pet $1 /$ hM3Dq was microinjected into the DR $(n=7)$, $b M 3 D q$ mRNA expression was detected in $78.4 \% \pm 1.5 \%$ of seroto- nergic neurons in the $\mathrm{DR}$, and $83.1 \% \pm 1.0 \%$ of $h M 3 D q^{+}$cells were also $\mathrm{TPH}^{+}$(Supplemental Figure 6A). No $h M 3 D q$ mRNA expression was detected in the area surrounding the DR. We administered saline or CNO i.p. into orexin/ataxin-3 mice injected with AAV-Pet1/hM3Dq in the DR immediately before the start of the dark period. Compared with saline administration, CNO substantially reduced the frequency of and time spent in cataplexy-like episodes during the subsequent 6 hours in the dark period (Figure 6 , A and B). REM sleep latency was significantly increased by $\mathrm{CNO}$ administration, whereas fragmentation of wakefulness was not improved (Figure 6, C and D).

Similarly, when AAV-PRSx8/hM3Dq was microinjected into the LC, $h M 3 D q$ mRNA expression was detected in $68.1 \% \pm 3.9 \%$ of noradrenergic neurons in the LC, and $81.1 \% \pm 1.5 \%$ of $h M 3 \mathrm{Dq}^{+}$ cells were also $\mathrm{TH}^{+}$(Supplemental Figure 6B). In these mice, the duration of wakefulness episodes during the subsequent 6 hours of the dark period was significantly increased, and the number of wakefulness episodes was significantly reduced by $\mathrm{CNO}$ versus saline treatment (Figure 6, G and $\mathrm{H}$ ). The total time spent in wakefulness during the same period was not significantly increased by $\mathrm{CNO}$ administration (Supplemental Figure 6B). Accordingly, 
A

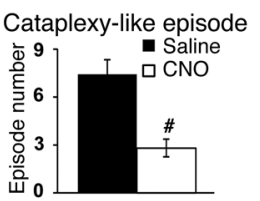

B

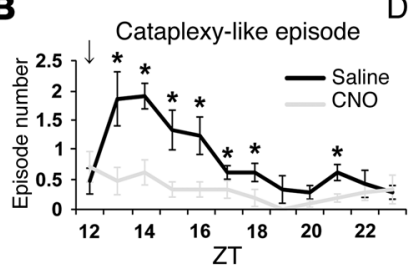

DR
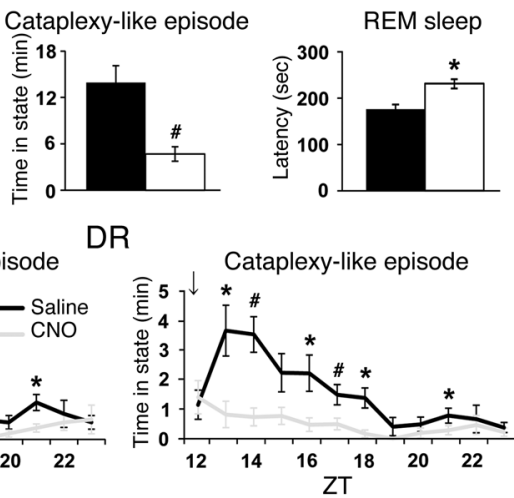

E

DR
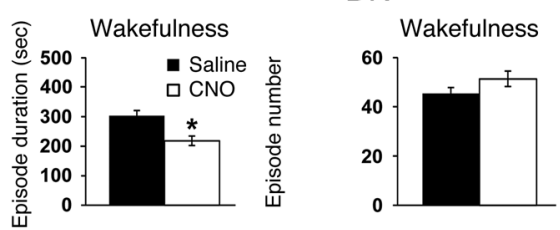

D

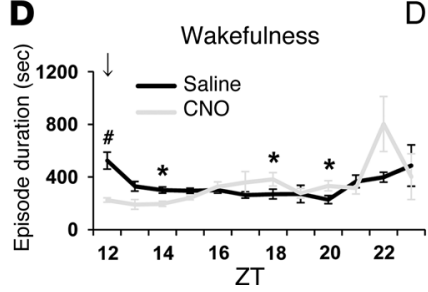

DR

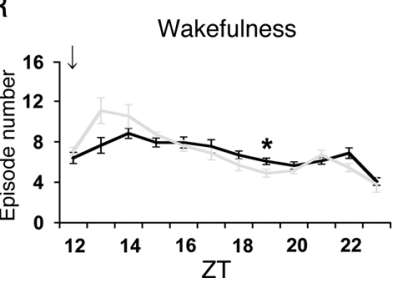

$\mathbf{F}$

G

H
LC
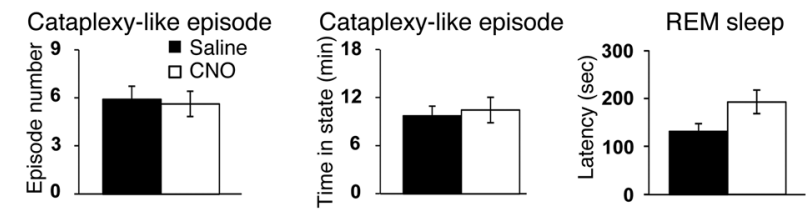

LC
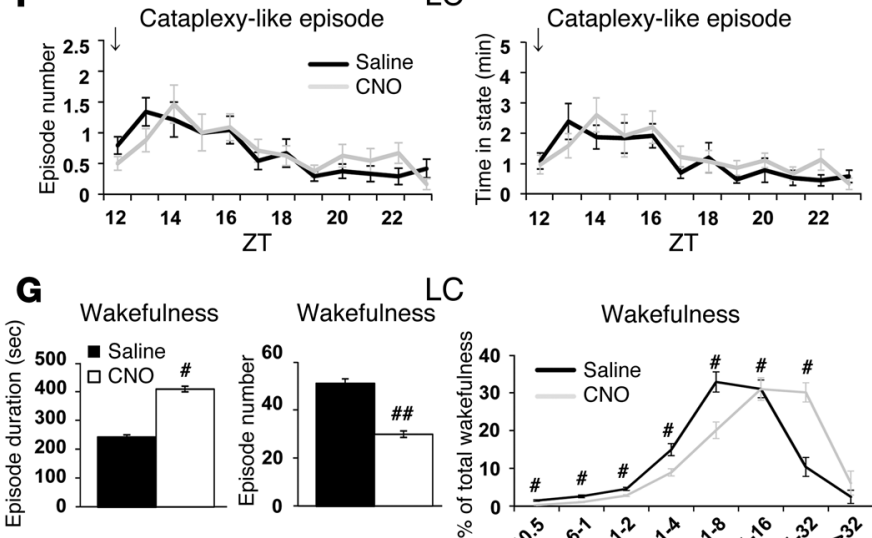

C
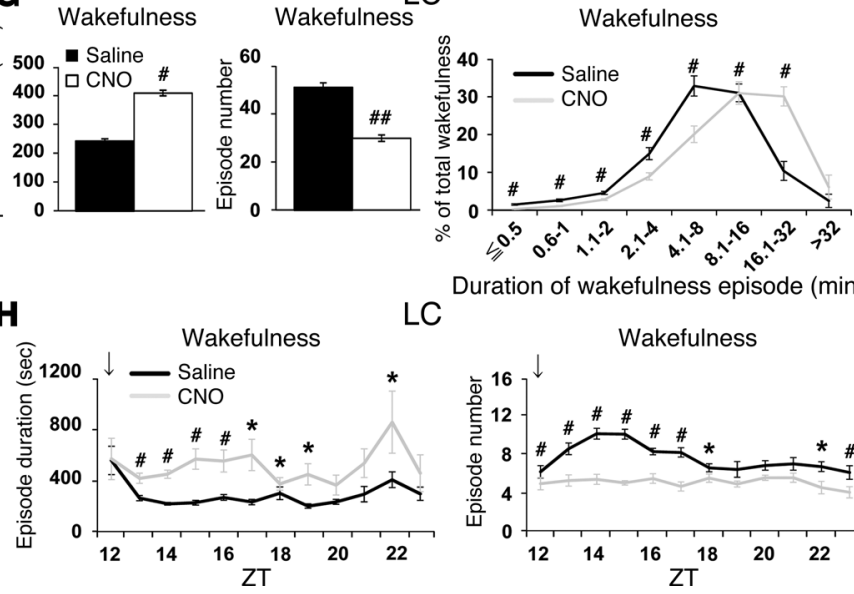
LC Duration of wakefulness episode (min)

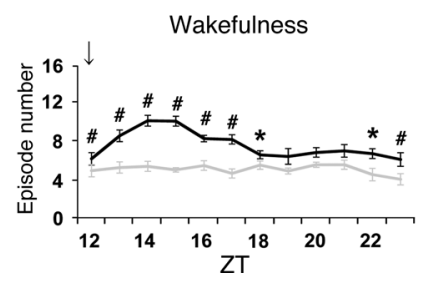

Figure 6

Pharmacogenetic activation of DR serotonergic and LC noradrenergic neurons suppresses cataplexy-like episodes and consolidates wakefulness, respectively. orexin/ataxin-3 mice with DR serotonergic neuron-selective (A-D; $n=7)$ or LC noradrenergic neuron-selective $(\mathbf{E}-\mathbf{H} ; n=8)$ expression of hM3Dq were injected with saline or CNO. Mean values of 3 nights' recordings are shown. (A and E) Number of and time spent in cataplexy-like episodes and REM sleep latency. (B and F) Hourly plots of number and total time of cataplexy-like episodes. (C and $\mathbf{G})$ Duration and number of wakefulness episodes within 12 hours after saline or CNO administration at ZT 12 (arrows). (G) Time-weighted frequency histogram of wakefulness duration is also shown $(P<0.001$, drug effect and interaction of drug and wakefulness duration, 2-way repeated-measures ANOVA). $\left(\mathbf{D}\right.$ and $\mathbf{H}$ ) Hourly plots of wakefulness duration and number within 12 hours after administration at ZT 12 (arrows). ${ }^{*} P<0.05,{ }^{\#} P<0.01,2$-tailed Student's paired $t$ test. Values are mean \pm SEM.

the proportion of wakefulness in long episodes was significantly increased in CNO- versus saline-treated mice (Figure 6G). In contrast, $\mathrm{CNO}$ administration did not significantly affect the frequency of cataplexy-like episodes (Figure 6, E and F). Importantly, the wake-stabilizing effect of CNO was not observed in orexin/ataxin-3 mice with off-target hM3Dq expression, which have similar numbers of hM3Dq-expressing cells in the regions surrounding the LC, but almost no such cells in the LC (Supplemental Figure 7, A-D). In addition, this effect of $\mathrm{CNO}$ was strongly correlated with the number of $h M 3 \mathrm{Dq}^{+} \mathrm{TH}^{+}$cells in the LC noradrenergic neurons, but not in any surrounding area (Supplemental Figure 7E).

Thus, these data suggest that pharmacogenetic activation of DR serotonergic and LC noradrenergic neurons in narcoleptic mice may be sufficient to substantially suppress cataplexy-like episodes and consolidate wakefulness, respectively. However, the possibility formally remains that a small number of non-serotonergic or nonnoradrenergic neurons in the DR or LC expressing hM3Dq also contribute to these effects.

\section{Discussion}

Technical considerations. One of the technical limitations of this study is that AAV vector-mediated expression of orexin receptors could not completely reproduce the endogenous expression patterns and levels driven by the native promoters (i.e., ectopic expression and overexpression). We used both ubiquitous and neuron type-selective promoters to drive orexin receptor expression. However, even with selective promoters, we observed considerable leaked expression. To address this problem, we performed a series of control injection studies and correlation analyses, which led to the same conclusion for multiple promoters of different characteristics: suppression of cataplexy-like episodes was correlated with the number of DR serotonergic neurons with OX2R restoration, while consolidation of fragmented wakefulness was correlated with the number of LC noradrenergic neurons with OX1R restoration. However, these results do not completely exclude the contributions of non-serotonergic and/or non-noradrenergic neurons around these areas. 
A

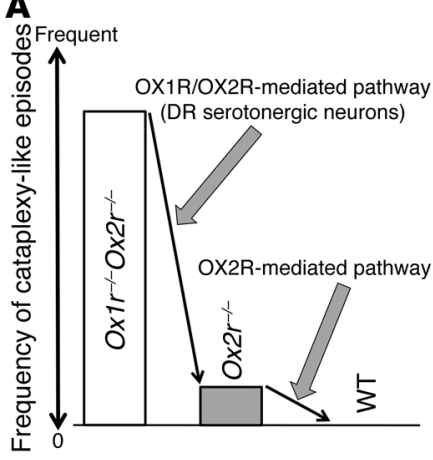

B

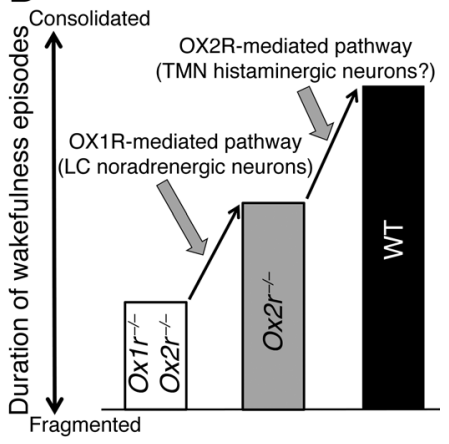

\section{Figure 7}

Proposed model for OX1R- and OX2R-mediated pathways in suppressing narcoleptic symptoms. (A) Prevention of cataplexy-like episodes. (B) Consolidation of wakefulness episodes.
It should be noted that the estimated specificities of Pet 1 and $P R S x 8$ promoters to serotonergic and noradrenergic neurons, respectively, were different between orexin receptor restoration experiments and DREADD experiments. This discrepancy seemed to result from the difference in the methods used to detect transgene expression. Orexin receptors were expressed as proteins fused to EGFP or EYFP, which are very stable, and they were detected by anti-GFP antibody with high sensitivity, likely leading to the efficient detection of leaked expression at very low levels. In contrast, because of the lack of a good antibody, $b M 3 D q$ expression was detected by in situ hybridization as mRNA, which is generally unstable, possibly missing low-level expression.

It is important to note that the orexin receptors in the restored cells might be expressed at levels different from those of the native receptors in WT mice. Therefore, from the standpoint of both patterns and expression levels, the reactivation approach, exemplified by Mochizuki et al. (30), combined with transgenic mice expressing Cre in cell type-specific manners (e.g., mice expressing Cre specifically in DR serotonergic neurons or in LC noradrenergic neurons) would be the ideal strategy to identify cells of physiological relevance in the true sense. Nevertheless, our approach seemed practical and still had several advantages, including higher throughput and detailed verification of receptor expression patterns owing to the EGFP and EYFP tags.

We failed to observe any amelioration of narcoleptic symptoms when orexin receptors were restored in the TMN, PPT, or PB. However, this result does not completely rule out the possible roles of orexin signaling in these nuclei in the suppression of narcolepsy; the possibility remains that more efficient restoration in these nuclei, in the number of restored cells, and/or in the expression levels within the individual cells would be able to suppress narcolepsy. Ectopic expression in the areas surrounding the TMN or PPT might also mask the contributions of TMN histaminergic and PPT cholinergic neurons. It is also possible that these nuclei play important roles as downstream targets of orexin neurons in cooperation with other nuclei, as discussed below for TMN histaminergic neurons. Similarly, although pharmacogenetic activation of $\mathrm{PB}$ neurons with leaked expression of hM3Dq did not enhance wakefulness in this study (Supplemental Figure 7, A-D), this result never denies the previously reported wake-promoting role of the $\mathrm{PB}(31)$.

Finally, our current study did not cover all populations of neurons implicated in the regulation of wakefulness and muscle tone. It thus does not exclude the potential roles in suppressing narcoleptic symptoms played by neurons that were not tested here. Nevertheless, we considered the nuclei examined in the current study to satisfy a set of candidates as the start point by which to dissect downstream targets of orexinergic modulation, according to well-characterized interactions between orexin neurons and monoaminergic and cholinergic neurons.

Roles of orexin receptor activation of $D R$ serotonergic neurons in the suppression of cataplexy-like episodes. Cataplexy-like episodes occurred frequently in $O x 1 r^{-1-} O x 2 r^{-1-}$ mice, but they rarely occurred in $O \times 2 r^{-/-}$mice and were absent in $O x 1 r^{-/-}$mice $(5,29,30)$, which suggests that disruption of both OX1R- and OX2R-mediated pathways is required for frequent cataplexy occurrence (Figure 7A). This notion is consistent with our conclusion that orexin signaling in DR serotonergic neurons may play a critical role in the prevention of cataplexy-like episodes, as most DR serotonergic neurons express both OX1R and OX2R (18). Both receptors couple to the $G_{q / 11}$ subclass of heterotrimeric $G$ proteins and evoke excitatory effects on neurons, suggestive of redundant intracellular signaling cascades triggered by OX1R and OX2R activation (1). It should be noted that nonselective restoration of OX2R in the DR tended to be more effective than serotonergicselective restoration. This outcome might stem from the difference in expression levels of restored OX2R driven by the different promoters. An alternative explanation implicates the presumable accessory roles of orexin signaling in non-serotonergic neurons of the DR (e.g., GABAergic neurons), although the number of nonserotonergic neurons with $\mathrm{OX} 2 \mathrm{R}$ restoration did not correlate with the extent of cataplexy-like episode suppression (Figure 3A).

The suppression of cataplexy-like episodes by DR serotonergic neurons was surprising, as LC noradrenergic neurons have been considered to be a likely candidate to prevent cataplexy, according to various pharmacological and electrophysiological studies. For example, cataplexy in humans and dogs is strongly suppressed by drugs that increase noradrenergic tone and is worsened by drugs that block noradrenergic signaling $(35,36)$. In addition, LC neurons cease firing during cataplexy in dogs (37). However, our observations never conflicted with the importance of the noradrenergic system in the pathophysiology of cataplexy. Rather, our results indicated that the activation of LC noradrenergic neurons by endogenous orexins is not sufficient to suppress cataplexy in narcoleptic mice. This view is consistent with the absence of cataplectic attacks in mice lacking $\operatorname{OX} 1 \mathrm{R}(1,5)$, the sole subtype expressed in LC noradrenergic neurons $(6,18)$. It is also likely that non-LC noradrenergic neurons play important roles in the suppression of cataplexy by pharmacological augmentation of systemic noradrenergic tone. This idea is supported by the facts that A1, A2, A5, and A7 neurons, rather than those of the LC, have been implicated in noradrenergic regulation of $\operatorname{REM}$ sleep $(22,38)$ 
and that A7 noradrenergic neurons send projections to the ventral horn of the spinal cord (26), which may be involved in the regulation of muscle tone. In any case, efficient anticataplectic effects of noradrenaline reuptake inhibitors may instead suggest that this effect is not dependent on the endogenous orexin system, as orexinergic activation of noradrenergic neurons is lacking in human narcolepsy patients, in which orexin neurons are degenerated. The logic here is analogous to the arousal effects of dopamine uptake inhibitors: although stimulants are effective in treating the sleepiness of narcolepsy by augmenting mesolimbic dopaminergic tone $(35,39)$, few researchers think that orexin neurons consolidate wakefulness mainly by activating dopaminergic neurons. Indeed, restoration of orexin signaling in the ventral tegmental area did not ameliorate the fragmentation of wakefulness in $\mathrm{O}_{1} \mathrm{r}^{-1-} \mathrm{O} \times 2 \mathrm{r}^{-1}$ mice (E. Hasegawa and M. Mieda, unpublished observations).

In contrast to LC noradrenergic neurons, DR serotonergic neurons do not completely cease discharging, but they greatly reduce firing rates, to an extent similar to those during NREM sleep (REM sleep-off serotonergic neurons) or REM sleep (REM sleep-reduced serotonergic neurons) in dogs (40). Thus, although changes in the discharge of LC noradrenergic neurons are more closely correlated with cataplexy than changes in DR serotonergic neuron discharge, these observations by themselves are not conclusive to demonstrate a causal relationship between firing rate and cataplexy occurrence. Suppression of DR serotonergic neurons by the focal application of a $5-\mathrm{HT}_{1 \mathrm{~A}}$ agonist was previously reported to induce cataplexylike episodes in cats, with no significant effect on REM sleep (41). Even if a reduction of DR serotonergic activity does not directly trigger cataplexy, the orexinergic activation of DR serotonergic neurons may prevent the permissive condition under which cataplexy can be induced by stimuli such as strong emotions. In this context, it is intriguing that we observed intense projections of DR serotonergic neurons to the amygdala (Supplemental Figure 8). Indeed, the amygdala contains cataplexy-related neurons (42), as well as REM-active neurons that are inhibited by DR stimulation (43). Thus, noradrenergic and DR serotonergic neurons may be involved in suppressing cataplexy at different levels.

Intriguingly, other aspects of REM sleep dysregulation in Ox1 $1 r^{-1-O} 2 r^{-1-}$ mice were not improved by DR serotonergic neuron-selective OX2R restoration, such as shortened REM sleep latency and excessive REM sleep time $(1,5)$. This outcome may reflect that REM sleep and cataplexy are regulated by at least partially distinct mechanisms, as suggested by previous reports and the fact that human narcolepsy patients usually remain conscious during cataplectic attacks $(2,41,44-46)$. Consistent with this view, DR serotonergic neurons do not cease firing during REM sleep without atonia after bilateral lesion of the mediodorsal pons (47), and they display suppression of spontaneous discharges during the atonic waking state induced by carbachol microinjection (48), which suggests that suppression of DR serotonergic neurons is correlated with REM atonia, rather than with REM sleep per se.

Roles of orexin receptor activation of $L C$ noradrenergic neurons in the consolidation of wakefulness. Whereas $\mathrm{O} \times 1 r^{-1}$ mice show almost normal sleep/wakefulness states (5), $O x 2 r^{-/-}$mice exhibit sleep/wake fragmentation (29). However, this fragmentation was less severe than that of orexin ${ }^{-1-}$ mice and $O \times 1 r^{-1-} \mathrm{O} \times 2 r^{-/-}$mice on the same C57BL/6J genetic background (Figure 5 and refs. 1, 30), which suggests that OX1R plays an important role in the maintenance of wakefulness in the absence of OX2R (18). Taken in conjunction with the prior report that LC noradrenergic neurons exclu- sively express OX1R in WT mice (18), our observations suggest that these neurons are responsible for the contribution of OX1R to the maintenance of wakefulness, while another OX2R-mediated mechanism is further required for the normal regulation of wakefulness duration (Figure 7B).

Although LC neurons are basically wake-on neurons, the role of LC noradrenergic neurons in arousal remained controversial. The view against their wake-promoting role was largely based on the fact that lesions limited to the LC only exert small effects on the amount of wakefulness (49). However, $\mathrm{Dbh}^{-/-}$mice, which lack endogenous noradrenaline, have been reported to exhibit reduced wakefulness as well as REM sleep (50). In addition, the LC-noradrenaline system was previously shown to be critical in maintaining the increased membrane potential of cortical neurons in wakeful versus sleep states (51). Moreover, recent optogenetic studies demonstrated a causal relationship between the firing of LC noradrenergic neurons and transitions from sleep to wakefulness (52) and further showed that inhibition of these neurons blocked the arousal effects of orexin neuron stimulation (53). These reports strongly support our conclusion about the role of the orexinergic regulation of LC noradrenergic neurons in the consolidation of wakefulness. A caveat to this view is that $O x 1 r^{-1}$ mice do not show any overt abnormality of sleep/wakefulness $(1,5)$. This discrepancy may be explained by the redundancy of networks comprising the arousal system and/or the rapid compensation of dysfunction of LC noradrenergic neurons, as lesions of the LC acutely reduce wakefulness for a few days, although the reduction dissipates rapidly with time (54-56). The presumptive compensation of OX1R deficiency in $\mathrm{Ox}_{1 r^{--}}$mice is further supported by the finding that acute pharmacological blockade of OX1R increases NREM sleep in rats (57). Thus, our strategy of focal orexin receptor restoration in Ox1 $r^{-1-} \mathrm{O} \times 2 r^{-1}$ mice provided important information that the lesion studies may not be able to demonstrate. However, rescue experiments may tend to overestimate the role of the restored component, owing to the lack of other redundant components. Therefore, our present results should be carefully interpreted in conjunction with those obtained using other approaches.

Mochizuki et al. previously reported that focal restoration of $\mathrm{OX} 2 \mathrm{R}$ in neurons of the posterior hypothalamus, including the TMN, reversed the fragmentation of wakefulness in $O x 2 r$-null $O \times 2 r^{T D}$ mice, in which a loxP-flanked transcription disrupter (TD) gene cassette prevents the expression of functional OX2R (30). In contrast, we failed to observe any improvement of fragmented wakefulness in $\mathrm{O} \times 1 r^{-1-} \mathrm{O} \times 2 r^{-1-}$ mice when OX2R expression was restored in the TMN and adjacent parts of the posterior hypothalamus. OX2R expression in the TMN may be sufficient to consolidate wakefulness only in the presence of OX1R expression, presumably in the LC (Figure 7B). Thus, OX2R receptors in histamine neurons are likely to be physiologically vital when OX1R levels are normal.

Does the neural circuit underlying narcolepsy differ between mice and dogs? At least 2 phenotypic forms of narcolepsy have been reported in canines. Familial narcolepsy has been maintained in lines of Doberman pinschers and Labrador retrievers and is now understood to be due to null mutations of the $O X 2 R$ gene, whereas sporadic nonfamilial narcolepsy is caused by deficiency of orexin peptides, like the majority of narcoleptic humans, and has been studied to a lesser extent in poodles, beagles, and other small breeds $(58,59)$. As the familial form of canine narcolepsy exhibits sleepiness and frequent cataplexy, OX1R has been considered to be dispensable for the underlying neural circuit pathophysiology 
of canine narcolepsy, apparently contradicting the results of our studies in mice. Thus, it is tempting to speculate a species difference with respect to orexin receptor expression, for example, that OX2R is the predominant subtype in the DR serotonergic neurons of dogs. Alternatively, the possibility remains that the neural mechanisms preventing narcolepsy are similar between dogs and mice, which depend on both OX1R and OX2R, as suggested by our present findings. The familial form of canine narcolepsy has been reported to be 30 - to 80 -fold less severely affected with cataplexy compared with the sporadic form (60). Unfortunately, no direct experimental comparison of sleepiness between the 2 canine models has been reported. The difference in genetic background and the limited availability of sporadically narcoleptic dogs make it difficult to precisely compare the severity of narcoleptic symptoms between familial (OX2R-deficient) and sporadic (orexin liganddeficient) canine narcolepsy. This drawback of canine studies may emphasize the importance of the use of mice in the current study, for which a larger number of animals with a uniform genetic background was available.

Conclusions. Here, we identified 2 critical efferent pathways of orexin neurons, which are most likely mediated by DR serotonergic and LC noradrenergic neurons differentially regulating cataplexylike episode prevention and wakefulness maintenance, respectively. Notably, complex neural circuits of the brainstem/hypothalamic glutamatergic and GABAergic neurons that generate/suppress wakefulness, NREM sleep, or REM sleep have recently emerged $(20,22,23,31,61)$. Orexin neurons, as well as monoaminergic and cholinergic neurons in the brainstem and hypothalamus, are likely to modulate those glutamatergic- and GABAergic-executing circuits of cortical activation and muscle atonia. Therefore, our findings provide new insight into not only the pathophysiology of narcolepsy, but also the mechanisms of the orexin system as a wakefulness stabilizer in normal sleep/wakefulness.

Finally, our success in improving narcoleptic symptoms by DREADD may lead to a novel type of gene therapy: an artificial receptor-ligand system (with well-established effectiveness, specificity, and safety) in which an artificial receptor can be delivered into neurons using a viral vector in order to ameliorate symptoms of interest, together with subsequent administration of an artificial ligand for the receptor with the appropriate timing.

\section{Methods}

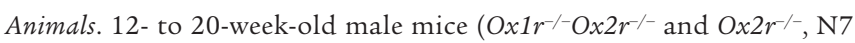
backcrossed to $\mathrm{C} 57 \mathrm{BL} / 6 \mathrm{~J}$, generated by crosses between homozygous mice; orexin/ataxin-3, N>7 backcrossed to C57BL/6J; and WT C57BL/6J) were used $(1,4,29)$. Mice were maintained under a strict 12 -hour light/12-hour dark cycle in a temperature- and humidity-controlled room and fed ad libitum.

Construction of recombinant $A A V$ vectors. The common structure of the AAV-2 ITR-containing plasmids used in this study is ITR-promotercDNA encoding a protein-WPRE-poly A signal-ITR. cDNAs encoding rat OX1R or OX2R tagged with EGFP or EYFP at the C terminus were used. The EF1 $\alpha$ promoter and ChR2-EYFP cDNA were derived from pAAV-DIO-hChR2(H134R)-EYFP-WPRE-pA, provided by K. Deisseroth (Stanford University, Stanford, California, USA). The CAG promoter was derived from $p A A V$-FLEX-rev-ChR2mCherry, provided by S.M. Sternson (Janelia Farm Research Campus, Ashbum, Virginia, USA). The PRS $x 8$ promoter fragment was provided by K.-S. Kim (Harvard Medical School, Belmont, Massachusetts, USA; ref. 25). For the Pet1 promoter, a 1.8-kb fragment containing a part of exon 1 and the $5^{\prime}$ flanking region of the mouse Pet1 gene (21) (residues $-1,675$ to +110 , with +1 the transcription initiation site; NCBI accession no. NT_039170) was amplified by PCR from genomic DNA of C57BL/6J. The HA-tagged $h M 3 D q$ cDNA was excised from the plasmid pcDNA5/FRT-HA- $h M 3 D q(33,34)$, provided by B.L. Roth (University of North Carolina, Chapel Hill, North Carolina, USA). AAV-2 vectors with a mutant form of the cap gene, provided by A. Srivastava (University of Florida, Gainesville, Florida, USA; ref. 62), were produced using a triple-transfection, helper-free method and purified as described previously (63). The titers of recombinant $\mathrm{AAV}$ vectors were as follows: $\mathrm{AAV}-\mathrm{EF} 1 \alpha / \mathrm{OX} 1 \mathrm{R}: \mathrm{EGFP}, 1.5 \times 10^{12}$ genome copies/ml; AAV-EF1 $\alpha / O X 2 \mathrm{R}:: E G F P, 1.7 \times 10^{12}$ genome copies $/ \mathrm{ml}$; AAV-CAG/EGFP, $3.2 \times 10^{12}$ genome copies/ml; AAV-PRSx8/OX1R::EYFP, $8.4 \times 10^{11}$ genome copies/ml; AAV-Pet1/OX2R::EYFP, $4.2 \times 10^{11}$ genome copies/ml; AAV-PRSx8/ChR2::EYFP, $5.0 \times 10^{12}$ genome copies/ml; AAV-Pet1/ ChR2::EYFP, $1.3 \times 10^{12}$ genome copies/ml; AAV-PRSx8/hM3Dq, $7.8 \times 10^{11}$ genome copies/ml; AAV-Pet1/hM3Dq, $1.1 \times 10^{12}$ genome copies $/ \mathrm{ml}$.

Surgery. Implantation of an EEG/EMG electrode and stereotaxic injection of AAV vectors were performed as described previously (63); 0.5-1.0 $\mu \mathrm{l}$ purified virus was delivered to each site $(0.05 \mu \mathrm{l} / \mathrm{min})$. After 5 minutes of rest, the needles were removed. The stereotaxic coordinates of the Hamilton needle syringe placement were as follows: LC (AP, $5.4 \mathrm{~mm}$; ML, $\pm 0.9 \mathrm{~mm}$ bilaterally; DV, $3.7 \mathrm{~mm}$ ); DR (AP, $4.4 \mathrm{~mm}$; ML, $\pm 0 \mathrm{~mm}$; DV, 3.0 and $3.5 \mathrm{~mm}$ ); TMN (AP, $2.5 \mathrm{~mm}$; ML, $\pm 1.0 \mathrm{~mm}$ bilaterally; DV, $5.6 \mathrm{~mm}$ ); PPT (AP, $4.5 \mathrm{~mm}$; $\mathrm{ML}, \pm 1.0 \mathrm{~mm}$ bilaterally; DV, $3.7 \mathrm{~mm}$ ); PB (AP, $-5.4 \mathrm{~mm}$; ML, $\pm 1.0 \mathrm{~mm}$ bilaterally; DV, $3.5 \mathrm{~mm}$ ). After surgery, all animals were housed individually for a recovery period of at least 14 days.

Sleep recordings. After the recovery period, EEG/EMG recordings were performed on 3 consecutive days, and EEG/EMG data were analyzed as previously described (63).

For some mice, we simultaneously videotaped the animals during the EEG/EMG recordings and scored cataplexy according to the criteria of Scammell et al. (19). As episodes identified as direct transitions from wakefulness to REM sleep on the EEG/EMG data perfectly correlated with episodes of abrupt immobility in the video data of these pilot recordings (total 77 episodes in 5 Ox1 $r^{/-O} 2 r^{/-}$mice; total 53 episodes in 2 orexin/ataxin-3 mice) and we needed to analyze many animals, we decided to record only the EEG/EMG for the rest of the mice studied. Although we believe that the identified episodes were equivalent to murine cataplexy, we carefully called the direct transitions from wakefulness to REM sleep cataplexy-like episodes, according to Scammell et al. (19).

EEG/EMG signals were scored in wakefulness, NREM sleep, REM sleep, or cataplexy-like episodes. REM sleep latency was calculated as the latency from the onset of sleep (transition from wakefulness to NREM sleep) to the first episode of REM sleep within that particular sleep episode. Sleep episodes without REM sleep were not included in the calculation of REM sleep latency.

Drugs and administration. CNO (C0832, Sigma-Aldrich) was dissolved in saline to a concentration of $0.5 \mathrm{mg} / \mathrm{ml}$. CNO or saline was administered by i.p. injection to each mouse $(0.3 \mathrm{ml} / 30 \mathrm{~g}$ body weight) at Zeitgeber time (ZT) 12. Each mouse received 3 saline and $3 \mathrm{CNO}$ administrations in an alternating manner, at intervals of 1 day after saline administration or 2 days after $\mathrm{CNO}$ administration.

Histological study. Double immunostaining was performed as previously described (64). Serial coronal brain sections ( $30 \mu \mathrm{m}$ thick) were collected in 4 series, 1 of which was further immunostained. EGFP/EYFP fluorescence was completely quenched before immunostaining by treating sections with $0.3 \%$ hydrogen peroxide. Antibodies used were as follows: goat anti-GFP (1:1,000; provided by M. Watanabe, Hokkaido University, Sapporo, Hokkaido, Japan); rabbit anti-TH (1:1,000; Chemicon); mouse antiTPH (1:200; Sigma-Aldrich); guinea pig anti-HDC (1:4,000; Biotechnik); goat anti-ChAT (1:2,000; Millipore); Alexa Fluor 488-conjugated donkey 
anti-rabbit IgG (1:500; Molecular Probes); Alexa Fluor 488-conjugated goat anti-guinea pig IgG (1:500; Molecular Probes); Alexa Fluor 594-conjugated donkey anti-goat IgG (1:500; Molecular Probes); and Alexa Fluor 594-conjugated goat anti-rabbit IgG (1:500; Molecular Probes). A representative optical section was imaged from each stained section by laser confocal microscopy (D-ECLIPSE C1si; Nikon), and fluorescent cells in the images were counted. These values did not provide accurate estimates of the total number of neurons, but were instead used to compare the extent of OX1R/OX2R restoration between cell groups. Double in situ hybridization to detect NPS and EYFP was performed as previously described (18) using 2 antisense riboprobes labeled with either fluorescein-UTP (Roche) (for EYFP, entire ORF; Clontech) or digoxygenin-UTP (Roche) (for NPS, 668-bp cDNA fragment of rat prepro-NPS containing $179 \mathrm{bp}$ of ORF and 490 bp of 3' UTR; provided by L. de Lecea, Stanford University, Stanford, California, USA). Fluorescent in situ hybridization combined with fluorescent immunostaining was performed essentially as described previously (65). Sections were processed first for in situ hybridization. This was done as described above, except that proteinase $\mathrm{K}$ was replaced by $1 \%$ sodium borohydride in PBS. Red fluorescent detection was performed using HNPP Fluorescent Detection Set (Roche) and digoxygenin-UTP-labeled riboprobe (full-length $h M 3 D q$ ) (34). Sections were then immunostained with rabbit anti-TH or mouse anti-TPH antibodies as described above.

Statistics. All results are expressed as mean \pm SEM. Changes in behavioral states caused by restoration of orexin receptors were analyzed by 1-way factorial ANOVA and Tukey-Kramer post-hoc tests. For time-weighted frequency histograms, data from every combination of 2 groups were analyzed by 2 -way repeated-measures ANOVA to detect significant interactions between wakefulness duration and orexin receptor expression, followed by post-hoc, 2-tailed Student's $t$ test (or, for DREADD experiments, post-hoc, 2-tailed Student's paired $t$ test). The effects of CNO administration in DREADD experiments were analyzed by 2-tailed Student's paired $t$ test. Hourly distributions of the parameters of wakefulness and cataplexy-like episodes were analyzed by 2 -way repeated-measures ANOVA to detect the significant effects of $\mathrm{CNO}$ administration or interactions between administration and time, followed by post-hoc, 2-tailed Student's paired $t$ test. To test the correlations between the number of cataplexy-like episodes or duration of wakefulness episodes and the number of OX2Ror OX1R-restored cells in each relevant area, Pearson $r$ and regression lines were calculated. $P$ values less than 0.05 were considered statistically significant. Only relevant information from the statistical analysis is indicated in the text and figures.

Study approval. All experimental procedures were approved by the appropriate institutional animal care and use committees of Kanazawa University.

\section{Acknowledgments}

This study was supported in part by Grants-in-Aid for Scientific Research (B) (grant no. 24390052) and for Challenging Exploratory Research (grant no. 23659134) from the Japan Society for the Promotion of Science (JSPS) (to M. Mieda); by the Mochida Memorial Foundation for Medical and Pharmaceutical Research (to M. Mieda); and by the Cabinet Office, Government of Japan, through its "Funding Program for Next Generation World-Leading Researchers" (grant no. LS048) (to T. Sakurai). The authors thank Karl Deisseroth for $p A A V$-DIO-bChR2(H134R)-EYFP-WPRE- $p A$; Scott M. Sternson for $p A A V$-FLEX-rev-ChR2mCherry; KwangSoo Kim for the PRSx8 promoter; Bryan L. Roth for pcDNA5/FRT-HA-bM3Dq; Masahiko Watanabe for the anti-GFP antibody; Arun Srivastava for $p A C G-2-Y 730 F$; and Luis de Lecea for rat NPS cDNA.

Received for publication May 13, 2013, and accepted in revised form October 15, 2013.

Address correspondence to: Michihiro Mieda, Department of Molecular Neuroscience and Integrative Physiology, Faculty of Medicine, Kanazawa University, 13-1 Takara-machi, Kanazawa, Ishikawa 920-8640, Japan. Phone: 81.76.265.2171; Fax: 81.76.234.4224; E-mail: mieda@med.kanazawa-u.ac.jp.
1. Sakurai T. The neural circuit of orexin (hypocretin): maintaining sleep and wakefulness. Nat Rev Neurosci. 2007;8(3):171-181.

2. Dauvilliers Y, Arnulf I, Mignot E. Narcolepsy with cataplexy. Lancet. 2007;369(9560):499-511.

3. Chemelli RM, et al. Narcolepsy in orexin knockout mice: molecular genetics of sleep regulation. Cell. 1999;98(4):437-451.

4. Hara J, et al. Genetic ablation of orexin neurons in mice results in narcolepsy, hypophagia, and obesity. Neuron. 2001;30(2):345-354.

5 . Hondo $\mathrm{M}$, et al. Histamine-1 receptor is not required as a downstream effector of orexin-2 receptor in maintenance of basal sleep/wake states. Acta Physiol (Oxf). 2010;198(3):287-294.

6. Bourgin $\mathrm{P}$, et al. Hypocretin-1 modulates rapid eye movement sleep through activation of locus coeruleus neurons. J Neurosci. 2000;20(20):7760-7765.

7. Huang ZL, et al. Arousal effect of orexin A depends on activation of the histaminergic system. Proc Natl Acad Sci U S A. 2001;98(17):9965-9970.

8. Xi MC, Morales FR, Chase MH. Effects on sleep and wakefulness of the injection of hypocretin-1 (orexin-A) into the laterodorsal tegmental nucleus of the cat. Brain Res. 2001;901(1-2):259-264.

9. Horvath TL, et al. Hypocretin (orexin) activation and synaptic innervation of the locus coeruleus noradrenergic system. J Comp Neurol. 1999; 415(2):145-159.

10. van den Pol AN, Ghosh PK, Liu RJ, Li Y, Aghajanian GK. Gao XB. Hypocretin (orexin) enhances neuron activity and cell synchrony in developing mouse GFP-expressing locus coeruleus. J Physiol. 2002; 541(pt 1):169-185.

11. Brown RE, Sergeeva O, Eriksson KS, Haas HL. Orexin A excites serotonergic neurons in the dorsal raphe nucleus of the rat. Neuropharmacology. 2001; 40(3):457-459.

12. Liu RJ, van den Pol AN, Aghajanian GK. Hypocretins (orexins) regulate serotonin neurons in the dorsal raphe nucleus by excitatory direct and inhibitory indirect actions. J Neurosci. 2002;22(21):9453-9464.

13. Bayer L, et al. Orexins (hypocretins) directly excite tuberomammillary neurons. Eur J Neurosci. 2001; 14(9):1571-1575.

14. Eriksson KS, Sergeeva O, Brown RE, Haas HL. Orexin/hypocretin excites the histaminergic neurons of the tuberomammillary nucleus. J Neurosci. 2001;21(23):9273-9279.

15. Yamanaka A, et al. Orexins activate histaminergic neurons via the orexin 2 receptor. Biochem Biophys Res Commun. 2002;290(4):1237-1245.

16. Burlet S, Tyler CJ, Leonard CS. Direct and indirect excitation of laterodorsal tegmental neurons by Hypocretin/Orexin peptides: implications for wakefulness and narcolepsy. J Neurosci. 2002; 22(7):2862-2872.

17. Marcus JN, et al. Differential expression of orexin receptors 1 and 2 in the rat brain.J Comp Neurol. 2001; 435(1):6-25.

18. Mieda M, Hasegawa E, Kisanuki YY, Sinton CM, Yanagisawa M, Sakurai T. Differential roles of orexin receptor-1 and -2 in the regulation of non-REM and REM sleep. J Neurosci. 2011;31(17):6518-6526.

19. Scammell TE, Willie JT, Guilleminault C, Siegel JM, International Working Group on Rodent Models of Narcolepsy. A consensus definition of cataplexy in mouse models of narcolepsy. Sleep. 2009; 32(1):111-116

20. Lu J, Sherman D, Devor M, Saper CB. A putative flipflop switch for control of REM sleep. Nature. 2006; 441(7093):589-594.

21. Scott MM, Krueger KC, Deneris ES. A differentially autoregulated Pet-1 enhancer region is a critical target of the transcriptional cascade that governs serotonin neuron development. J Neurosci. 2005; 25(10):2628-2636.

22. Luppi $\mathrm{PH}$, et al. The neuronal network responsible for paradoxical sleep and its dysfunctions causing narcolepsy and rapid eye movement (REM) behavior disorder. Sleep Med Rev. 2011;15(3):153-163.

23. Vetrivelan R, Chang C, Lu J. Muscle tone regulation during REM sleep: neural circuitry and clinical significance. Arch Ital Biol. 2011;149(4):348-366.

24. Lu J, Jhou TC, Saper CB. Identification of wakeactive dopaminergic neurons in the ventral periaqueductal gray matter. J Neurosci. 2006;26(1):193-202.

25. Hwang DY, Carlezon WA Jr, Isacson O, Kim KS. A high-efficiency synthetic promoter that drives transgene expression selectively in noradrenergic neurons. Hum Gene Ther. 2001;12(14):1731-1740.

26. Bruinstroop E, et al. Spinal projections of the A5, A6 (locus coeruleus), and A7 noradrenergic cell groups in rats. J Comp Neurol. 2012;520(9):1985-2001.

27. Card JP, et al. Microdissection of neural networks by conditional reporter expression from a Brainbow herpesvirus. Proc Natl Acad Sci U S A. 2011; 108(8):3377-3382.

28. Card JP, Sved JC, Craig B, Raizada M, Vazquez J, 
Sved AF. Efferent projections of rat rostroventrolateral medulla C1 catecholamine neurons: Implications for the central control of cardiovascular regulation. J Comp Neurol. 2006;499(5):840-859.

29. Willie JT, et al. Distinct narcolepsy syndromes in Orexin receptor- 2 and Orexin null mice: molecular genetic dissection of Non-REM and REM sleep regulatory processes. Neuron. 2003;38(5):715-730.

30. Mochizuki T, et al. Orexin receptor 2 expression in the posterior hypothalamus rescues sleepiness in narcoleptic mice. Proc Natl Acad Sci U S A. 2011; 108(11):4471-4476.

31. Fuller PM, Sherman D, Pedersen NP, Saper CB, Lu J. Reassessment of the structural basis of the ascending arousal system. J Neurosci. 2011; 519(5):933-956.

32. Xu YL, et al. Neuropeptide S: a neuropeptide promoting arousal and anxiolytic-like effects. Neuron. 2004;43(4):487-497.

33. Alexander GM, et al. Remote control of neuronal activity in transgenic mice expressing evolved $G$ protein-coupled receptors. Neuron. 2009;63(1):27-39.

34. Armbruster BN, Li X, Pausch MH, Herlitze S, Roth BL. Evolving the lock to fit the key to create a family of $\mathrm{G}$ protein-coupled receptors potently activated by an inert ligand. Proc Natl Acad Sci U S A. 2007; 104(12):5163-5168.

35. Hirai N, Nishino S. Recent advances in the treatment of narcolepsy. Curr Treat Options Neurol. 2011; 13(5):437-457.

36. Nishino S, Mignot E. Pharmacological aspects of human and canine narcolepsy. Prog Neurobiol. 1997;52(1):27-78.

37. Wu MF, Gulyani SA, Yau E, Mignot E, Phan B, Siegel JM. Locus coeruleus neurons: cessation of activity during cataplexy. Neuroscience. 1999; 91(4):1389-1399.

38. Léger L, Goutagny R, Sapin E, Salvert D, Fort P, Luppi PH. Noradrenergic neurons expressing Fos during waking and paradoxical sleep deprivation in the rat. J Chem Neuroanat. 2009;37(3):149-157.

39. Lazarus M, Huang ZL, Lu J, Urade Y, Chen JF. How do the basal ganglia regulate sleep-wake behavior? Trends Neurosci. 2012;35(12):723-732.

40. Wu MF, John J, Boehmer LN, Yau D, Nguyen GB, Siegel JM. Activity of dorsal raphe cells across the sleep-waking cycle and during cataplexy in narcoleptic dogs. J Physiol. 2004;554(pt 1):202-215.
41. Sakai K, Crochet S. Role of dorsal raphe neurons in paradoxical sleep generation in the cat: no evidence for a serotonergic mechanism. EurJ Neurosci. 2001; 13(1):103-112.

42. Gulyani S, Wu MF, Nienhuis R, John J, Siegel JM. Cataplexy-related neurons in the amygdala of the narcoleptic dog. Neuroscience. 2002;112(2):355-365.

43. Jha SK, Ross RJ, Morrison AR. Sleep-related neurons in the central nucleus of the amygdala of rats and their modulation by the dorsal raphe nucleus. Physiol Behav. 2005;86(4):415-426.

44. Burgess CR, Tse G, Gillis L, Peever JH. Dopaminergic regulation of sleep and cataplexy in a murine model of narcolepsy. Sleep. 2010;33(10):1295-1304.

45. John J, Wu MF, Boehmer LN, Siegel JM. Cataplexyactive neurons in the hypothalamus: implications for the role of histamine in sleep and waking behavior. Neuron. 2004;42(4):619-634.

46. Okura M, Riehl J, Mignot E, Nishino S. Sulpiride, a D2/D3 blocker, reduces cataplexy but not REM sleep in canine narcolepsy. Neuropsychopharmacology. 2000;23(5):528-538.

47. Trulson ME, Jacobs BL, Morrison AR. Raphe unit activity during REM sleep in normal cats and in pontine lesioned cats displaying REM sleep without atonia. Brain Res. 1981;226(1-2):75-91.

48. Steinfels GF, Heym J, Strecker RE, Jacobs BL. Raphe unit activity in freely moving cats is altered by manipulations of central but not peripheral motor systems. Brain Res. 1983;279(1-2):77-84

49. Gompf HS, et al. Locus ceruleus and anterior cingulate cortex sustain wakefulness in a novel environment. J Neurosci. 2010;30(43):14543-14551.

50. Ouyang M, Hellman K, Abel T, Thomas SA. Adrenergic signaling plays a critical role in the maintenance of waking and in the regulation of REM sleep. J Neurophysiol. 2004;92(4):2071-2082.

51. Constantinople CM, Bruno RM. Effects and mechanisms of wakefulness on local cortical networks. Neuron. 2011;69(6):1061-1068.

52. Carter ME, et al. Tuning arousal with optogenetic modulation of locus coeruleus neurons. Nat Neurosci. 2010;13(12):1526-1533.

53. Carter ME, Brill J, Bonnavion P, Huguenard JR, Huerta $\mathrm{R}$, de Lecea L. Mechanism for Hypocretin-mediated sleep-to-wake transitions. Proc Natl Acad Sci US A. 2012;109(39):E2635-E2644.

54. González MM, Debilly G, Valatx JL. Noradrenaline neurotoxin DSP-4 effects on sleep and brain temperature in the rat. Neurosci Lett. 1998;248(2):93-96.

55. Jones BE, Harper ST, Halaris AE. Effects of locus coeruleus lesions upon cerebral monoamine content, sleep-wakefulness states and the response to amphetamine in the cat. Brain Res. 1977; 124(3):473-496

56. Monti JM, D’Angelo L, Jantos H, Barbeito L, Abó V. Effect of DSP-4, a noradrenergic neurotoxin, on sleep and wakefulness and sensitivity to drugs acting on adrenergic receptors in the rat. Sleep. 1988; 11(4):370-377.

57. Morairty SR, et al. Dual hypocretin receptor antagonism is more effective for sleep promotion than antagonism of either receptor alone. PloS One. 2012; 7(7):e39131.

58. Lin L, et al. The sleep disorder canine narcolepsy is caused by a mutation in the hypocretin (orexin) receptor 2 gene. Cell. 1999;98(3):365-376

59. Ripley B, Fujiki N, Okura M, Mignot E, Nishino S. Hypocretin levels in sporadic and familial cases of canine narcolepsy. Neurobiol Dis. 2001;8(3):525-534.

60. Baker TL, Foutz AS, McNerney V, Mitler MM, Dement WC. Canine model of narcolepsy: genetic and developmental determinants. Exp Neurol. 1982; 75(3):729-742.

61. Anaclet $\mathrm{C}$, et al. Identification and characterization of a sleep-active cell group in the rostral medullary brainstem. J Neurosci. 2012;32(50):17970-17976.

62. Zhong L, et al. Next generation of adeno-associated virus 2 vectors: point mutations in tyrosines lead to high-efficiency transduction at lower doses. Proc Natl Acad Sci U S A. 2008;105(22):7827-7832.

63. Sasaki K, Suzuki M, Mieda M, Tsujino N, Roth B, Sakurai T. Pharmacogenetic modulation of orexin neurons alters sleep/wakefulness states in mice. PloS One. 2011;6(5):e20360.

64. Mieda M, Williams SC, Sinton CM, Richardson JA, Sakurai T, Yanagisawa M. Orexin neurons function in an efferent pathway of a food-entrainable circadian oscillator in eliciting food-anticipatory activity and wakefulness. J Neurosci. 2004; 24(46):10493-10501.

65. Mieda M, Williams SC, Richardson JA, Tanaka $\mathrm{K}$, Yanagisawa M. The dorsomedial hypothalamic nucleus as a putative food-entrainable circadian pacemaker. Proc Natl Acad Sci U S A. 2006; 103(32):12150-12155. 